\title{
PERK activation by SB202190 ameliorates amyloidogenesis via the TFEB-induced autophagy-lysosomal pathway
}

\author{
Mihyang Do ${ }^{1,}{ }^{,}$, Jeongmin Park ${ }^{1,}{ }^{*}$, Yubing Chen ${ }^{1}$, So-Young Rah ${ }^{2}$, Thu-Hang Thi Nghiem ${ }^{1}$, \\ Jeong Heon Gong ${ }^{1}$, Seong-A Ju${ }^{1}$, Byung-Sam Kim${ }^{1}$, Rina $\mathrm{Yu}^{3}$, Jeong Woo Park ${ }^{1}$, Stefan W. Ryter ${ }^{4}$, \\ Young-Joon Surh ${ }^{5,6}$, Uh-Hyun Kim², Yeonsoo Joe ${ }^{1}$, Hun Taeg Chung ${ }^{1}$ \\ ${ }^{1}$ Department of Biological Sciences, University of Ulsan, Ulsan 44610, Republic of Korea \\ ${ }^{2}$ National Creative Research Laboratory for $\mathrm{Ca}^{2+}$ Signaling Network, Chonbuk National University Medical School, \\ Jeonju 54907, Republic of Korea \\ ${ }^{3}$ Department of Food Science and Nutrition, University of Ulsan, Ulsan 44610, Republic of Korea \\ ${ }^{4}$ Department of Medicine, Weill Cornell Medicine, New York, NY 10065, USA \\ ${ }^{5}$ Department of Molecular Medicine and Biopharmaceutical Sciences, Graduate School of Convergence Science \\ and Technology, Seoul National University, Seoul 08826, Republic of Korea \\ ${ }^{6}$ Cancer Research Institute, Seoul National University, Seoul 03080, Republic of Korea \\ *Equal contribution
}

Correspondence to: Yeonsoo Joe, Hun Taeg Chung; email: jcantibody@ulsan.ac.kr, chung@ulsan.ac.kr Keywords: amyloidogenesis, mitochondrial reactive oxygen species, PERK, transcription factor-EB, autophagy-lysosome pathway Received: August 13, 2021

Accepted: February 8, 2022

Published: February 15, 2022

Copyright: (C) 2022 Do et al. This is an open access article distributed under the terms of the Creative Commons Attribution License (CC BY 3.0), which permits unrestricted use, distribution, and reproduction in any medium, provided the original author and source are credited.

\section{ABSTRACT}

The protein kinase $R$ (PKR)-like endoplasmic reticulum (ER) kinase (PERK), a key ER stress sensor of the unfolded protein response (UPR), can confer beneficial effects by facilitating the removal of cytosolic aggregates through the autophagy-lysosome pathway (ALP). In neurodegenerative diseases, the ALP ameliorates the accumulation of intracellular protein aggregates in the brain. Transcription factor-EB (TFEB), a master regulator of the ALP, positively regulates key genes involved in the cellular degradative pathway. However, in neurons, the role of PERK activation in mitigating amyloidogenesis by ALP remains unclear. In this study, we found that SB202190 selectively activates PERK independently of its inhibition of p38 mitogen-activated protein kinase, but not inositol-requiring transmembrane kinase/endoribonuclease-1 $\alpha$ (IRE1 $\alpha$ ) or activating transcription factor 6 (ATF6), in human neuroblastoma cells. PERK activation by SB202190 was dependent on mitochondrial ROS production and promoted $\mathrm{Ca}^{2+}$-calcineurin activation. The activation of the PERK-Ca ${ }^{2+}$-calcineurin axis by SB202190 positively affects TFEB activity to increase ALP in neuroblastoma cells. Collectively, our study reveals a novel physiological mechanism underlying ALP activation, dependent on PERK activation, for ameliorating amyloidogenesis in neurodegenerative diseases.

\section{INTRODUCTION}

Amyloids are a highly organized form of protein aggregation typically associated with human neuropathies, including Alzheimer's diseases (AD), Parkinson's diseases (PD), and Huntington's diseases (HD) [1]. Under various pathological conditions, amyloid fibrils arise from amyloidogenesis as a bio- chemical process in which a soluble protein is converted into insoluble and fibrillar protein aggregates $[2,3]$. Accumulated aggregated proteins, such as amyloid $\beta$ $(\mathrm{A} \beta)$, can trigger endoplasmic reticulum (ER) stress and lead to many neurodegenerative diseases [4-7]. ER stress occurring in neurodegenerative diseases activates all three pathways of the mammalian unfolded protein response (UPR), each represented by its unique UPR 
sensor: PKR-like ER kinase (PERK) [8], inositolrequiring transmembrane kinase/endoribonuclease- $1 \alpha$ (IRE1 $\alpha$ ) [9] and activating transcription factor 6 (ATF6) [10]. However, it has become evident that the PERK pathway exerts a major role in the resolution of aggregated protein cytotoxicity. PERK activation by ER stress mitigates cerebral $A \beta$ accumulation by reducing $\beta$-secretase-1 levels and cognition deficits in $\mathrm{AD}[11$, 12]. Moreover, UPR induction leads to the accumulation $\alpha$-synuclein ( $\alpha$-syn) that contributes to neurodegeneration in $\alpha$-synucleinopathies [13]. Beyond its canonical role in the UPR, PERK as a structural tether of the mitochondria-associated ER membrane (MAMs), serves as a physical and functional connection between the ER and the mitochondria [14, 15]. PERK signaling facilitates $\mathrm{Ca}^{2+}$ efflux from the ER, and increases mitochondrial $\mathrm{Ca}^{2+}$ levels, leading to increased generation of mtROS [16]. The increase of cytosolic $\mathrm{Ca}^{2+}$ levels by PERK [16] activates calcineurin, a $\mathrm{Ca}^{2+}$ and calmodulin-dependent phosphatase [17]. In particular, the interaction of PERK and calcineurin in astrocytes promotes cell survival after acute brain injuries [18].

Neurodegenerative disease is strongly associated with defects in the autophagy-lysosomal pathway (ALP) [19]. Moreover, the clearance of intracellular aggregates in the brain typically improves symptoms of neurodegenerative diseases such as $\mathrm{AD}, \mathrm{PD}$, and $\mathrm{HD}$ $[20,21]$. ALP has been widely demonstrated to ameliorate pathology in these diseases [22-24]. TFEB, a global regulator of ALP, is a basic helix-loop-helix leucine zipper transcription factor of the MiT/TFE family [25]. TFEB binds a promoter motif responsible for coordinating the expression of lysosomal genes; identified as the coordinated lysosomal expression and regulation (CLEAR) element [26]. In addition, TFEB regulates autophagy through enhancing autophagosome formation and autophagosome-lysosome fusion [27]. Stress conditions, such as starvation or exposure to ER stress-inducing agents, result in translocation of TFEB to the nucleus, where it promotes transcription of its target genes [26, 27]. TFEB nuclear translocation is associated with its phosphorylation state [27]. The increase of $\mathrm{Ca}^{2+}$ levels lead to the activation of the phosphatase calcineurin, which dephosphorylates TFEB, resulting in TFEB nuclear translocation and the transcription of target genes [28]. Enhancing the ALP through TFEB overexpression has marked beneficial effects in ameliorating amyloidogenesis in neurodegenerative diseases $[22,23,26]$.

We demonstrate here that PERK activation can mitigate amyloidogenesis through promoting the ALP. SB202190, previously identified as a p38 MAPK inhibitor, increases the TFEB-dependent ALP via $\mathrm{Ca}^{2+}$ dependent calcineurin activation independent of p38 MAPK inhibition [29]. We describe a new function of SB202190, demonstrating that this molecule can activate PERK through mtROS production leading to the release of $\mathrm{Ca}^{2+}$ from the ER in human neuroblastoma cells. SB202190-induced PERK activation enhances TFEB nuclear translocation, leading to activation of the ALP. Finally, we demonstrate that PERK activation, as a novel target of SB202190, promotes the degradation of APP or $\alpha$-syn accumulation via ALP in SH-SY5Y cells. These studies confirm the feasibility of PERK pathway targeting as a therapeutic approach for neurodegenerative diseases.

\section{RESULTS}

\section{SB202190 activates the PERK/eIF2 $\alpha / A T F 4$ pathway}

Our previous data have suggested that PERK activation can promote calcineurin-dependent TFEB nuclear translocation, and subsequent increases in autophagy and lysosomal-related gene expression [30]. A recent study has found that, among several known p38 MAPK inhibitors, only SB202190 can promote autophagy and lysosomal biogenesis [29]. These effects were dependent on calcineurin rather than via the inhibition of p38 MAPK [29]. In this study, we investigated the mechanisms by which SB202190 can induce autophagy and lysosomal biogenesis, and the underlying role of $\mathrm{Ca}^{2+}$ and PERK signaling in the response. We first assessed whether SB202190 activates PERK in various cells. SB202190 increased PERK activation in a dosedependent manner in HEK293 cells (Figure 1A). We also confirmed that the activation of PERK by SB202190 was independent of p38 MAPK, by using siRNA targeting p38 MAPK (si-p38 MAPK). PERK activation by SB202190 or thapsigargin (Tg), an inhibitor of ER stress, was similar in both the scrambled control RNA (scRNA)-transfected and si-p38 MAPKtransfected SH-SY5Y cells (Supplementary Figure 1). In addition, PERK as well as its downstream targets, eIF2 $\alpha$ and ATF4, were activated by SB202190 treatment. This observation was similar to the effect elicited by $\mathrm{Tg}$, an inhibitor of the ER $\mathrm{Ca}^{2+}$-ATPase, used as a positive control (Figure 1B). Tg induces a higher degree of phosphorylation of PERK than elicited by SB202190, which results in an apparent upward shift in the mobility of the PERK band. To confirm the requirement of PERK activation in the downstream effects of SB202190 treatment, including eIF2 $\alpha$ and ATF4 activation, HEK293 cells were pretreated with a PERK inhibitor (GSK2606414). The inhibition of PERK with GSK2606414 attenuated the activation of eIF2 $\alpha$ and ATF4 in response to SB202190 (Figure 1B). To investigate the effects of PERK activation in neuronal cells, the human neuroblastoma cell line SH- 
SY5Y was treated with SB202190. We found that SB202190 activated the PERK signaling pathway in control siRNA-transfected cells, but not in SH-SY5Y cells subjected to siRNA-mediated PERK knockdown (Figure 1C). Consistent with observations in SH-SY5Y cells, treatment with SB202190 induced the activation of the PERK pathway in MEF cells isolated from
Perk $^{+/+}$mice, but not Perk $^{-/}$mice (Figure 1D). To assess whether SB202190 can activate the other branches of the UPR (i.e., IRE1 $\alpha$ and ATF6), we analyzed the levels of Xbp1 splicing and GRP78 expression in SB202190-treated SH-SY5Y cells. Both Xbp1 splicing and GRP78 expression were not affected by SB202190 treatment but were responsive to $\mathrm{Tg}$
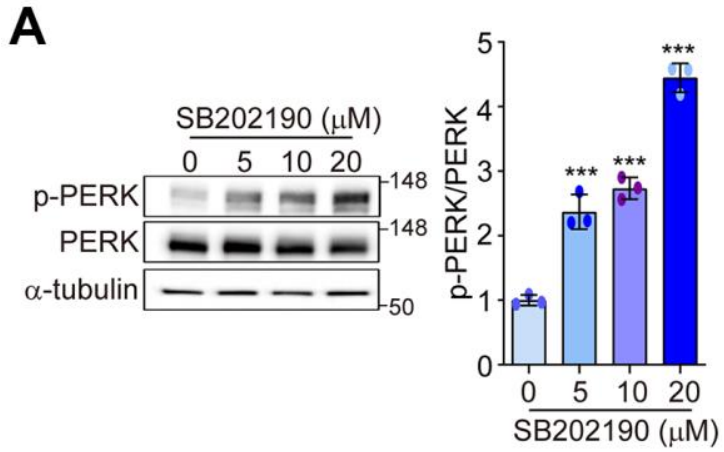

B
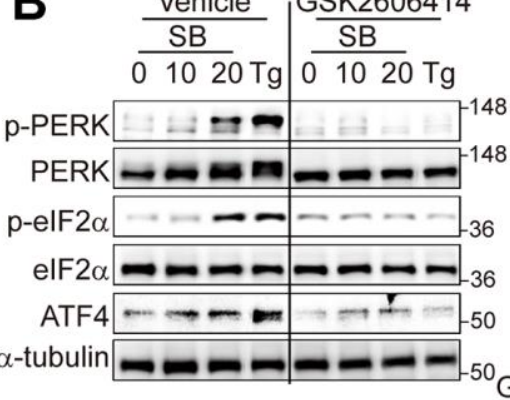

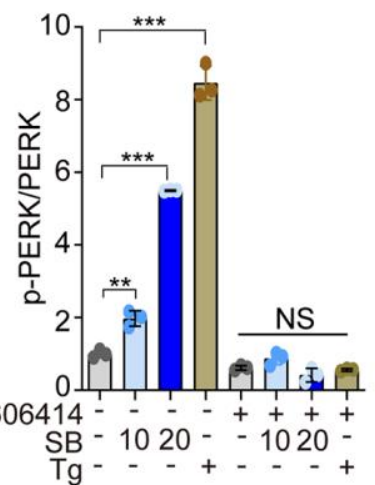

E
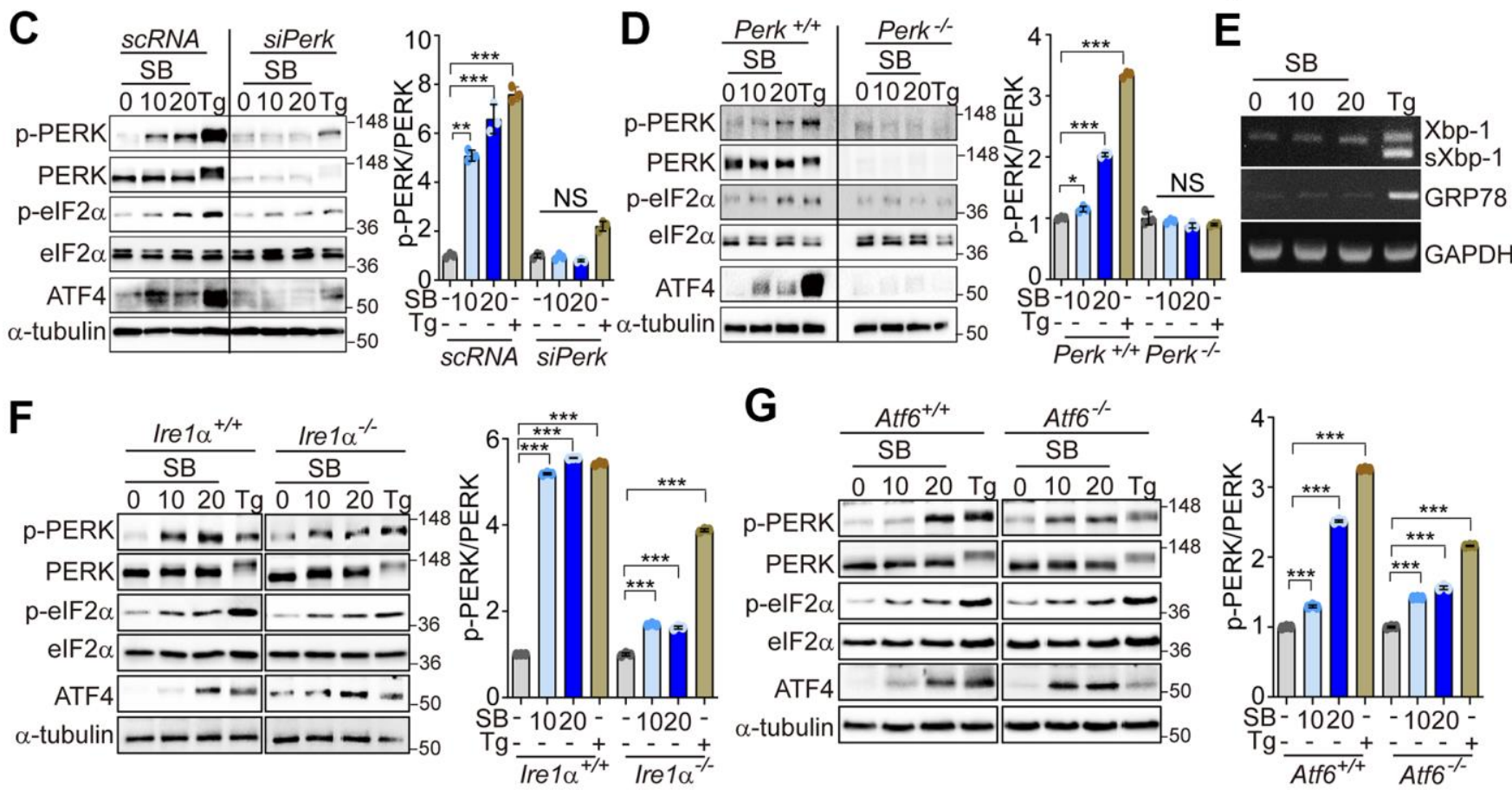

Figure 1. SB202190 activates the PERK/elF2 $\alpha$ /ATF4 pathway. (A) HEK293 cells were treated with SB202190 (0, 5, 10, and $20 \mu \mathrm{M})$ for 6 h. PERK phosphorylation was determined by western blotting. Quantification of p-PERK is shown in the right panel. (B) HEK293 cells were incubated with SB202190 (10 and $20 \mu \mathrm{M})$ for $6 \mathrm{~h}$ after pretreatment with or without the PERK inhibitor, GSK2606414 (1 $\mu \mathrm{M})$ for $1 \mathrm{~h}$. Thapsigargin (Tg, $2 \mu \mathrm{M}$ ) was used as a positive control. Cell lysates were used for western blotting analysis for $p$-PERK, PERK, p-elF2 $\alpha$, elF2 $\alpha$, and ATF4. (C) For knockdown of Perk, SH-SY5Y cells were transfected with control siRNA (scRNA) or siPerk for $48 \mathrm{~h}$ and then treated with different doses $(10$ and $20 \mu \mathrm{M})$ of SB202190 (SB) or $\operatorname{Tg}(2 \mu \mathrm{M})$ for $6 \mathrm{~h}$. Cell lysates were measured for PERK activation by western blot using the indicated antibodies. (D) Perk ${ }^{+/+}$and Perk ${ }^{-}$MEFs were treated with SB202190 (10 and $\left.20 \mu \mathrm{M}\right)$ for $6 \mathrm{~h}$ or Tg $(2 \mu \mathrm{M})$. The levels of p-PERK, PERK, p-elF2 $\alpha$, elF2 $\alpha$, and ATF4 were measured by western blotting. (E) SH-SY5Y cells were treated with SB202190 (10 and $20 \mu \mathrm{M})$ or Tg ( 2 $\mu \mathrm{M})$ for 6 h. Xbp-1 splicing and Grp78 expression were detected by RT-PCR. (F, G) Hepatocytes isolated from Ire1 $\alpha^{+/}$, Ire1 $\alpha^{\%}(\mathbf{F})$ or Atf6 $\alpha^{+/+}$, and Atf6 $\%(\mathbf{G})$ mice were treated with SB202190 $(10$ and $20 \mu \mathrm{M})$ for $6 \mathrm{~h}$ to assess the levels of PERK phosphorylation and ATF4 expression by western blotting. Quantification of p-PERK is shown in the right panel (A-D, F, G). Data are mean \pm SD $(n=3) ;{ }^{*} p<0.05, * * p<0.01$, and $* * * p<0.001$. 
treatment (Figure 1E). The activation of the PERK pathway by SB202190 was not affected by genetic deficiency of IRE1 $\alpha$ or ATF6 (Figure 1F, 1G). These results demonstrate that SB202190 can selectively induce activation of the PERK branch of the UPR, but not the IRE1 $\alpha$ or ATF6 branches of the UPR.

\section{PERK activation with SB202190 is induced by mtROS production}

Low levels of mtROS can selectively activate the PERK/eIF2 $\alpha /$ ATF4 axis to preserve cellular homeostasis [30-33]. Modulation of the PERK pathway may protect against neurodegeneration [7]. Thus, we first investigated whether SB202190-induced PERK

A

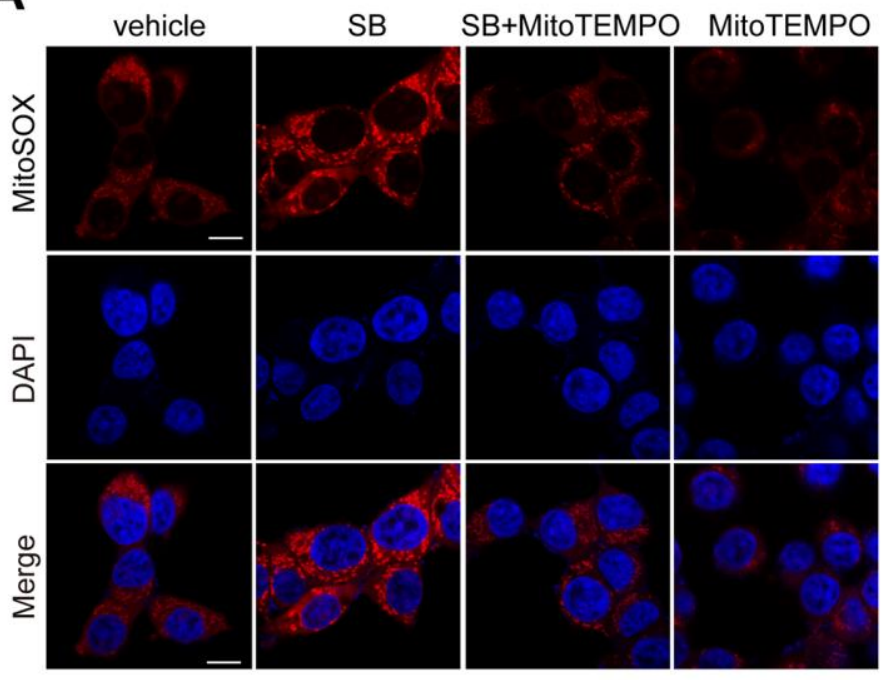

C
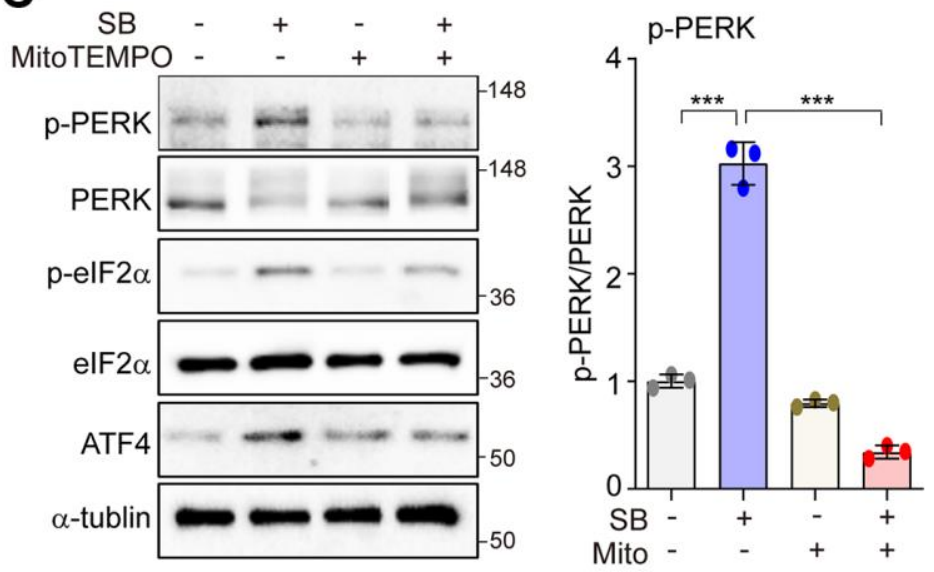

activation was mediated by mtROS in human neuronal cells. In SH-SY5Y cells, treatment with SB202190 enhanced mtROS levels as detected using the mitochondrial superoxide indicator MitoSOX and analyzed by either confocal microscopy (Figure 2A) and FACS (Figure 2B). Conversely, SB202190 induced mtROS levels were diminished in cells treated with MitoTEMPO, an mtROS scavenger (Figure 2A 2B). In accordance with observations in SH-SY5Y cells, we confirmed that SB202190 enhanced the generation of mtROS in HEK293 cells (Supplementary Figure 2A). To determine whether SB202190 treatment activates the PERK pathway via mtROS, the levels of phosphorylation of PERK, eIF2 $\alpha$, and ATF4 were measured in MitoTEMPO-treated SH-SY5Y and

B
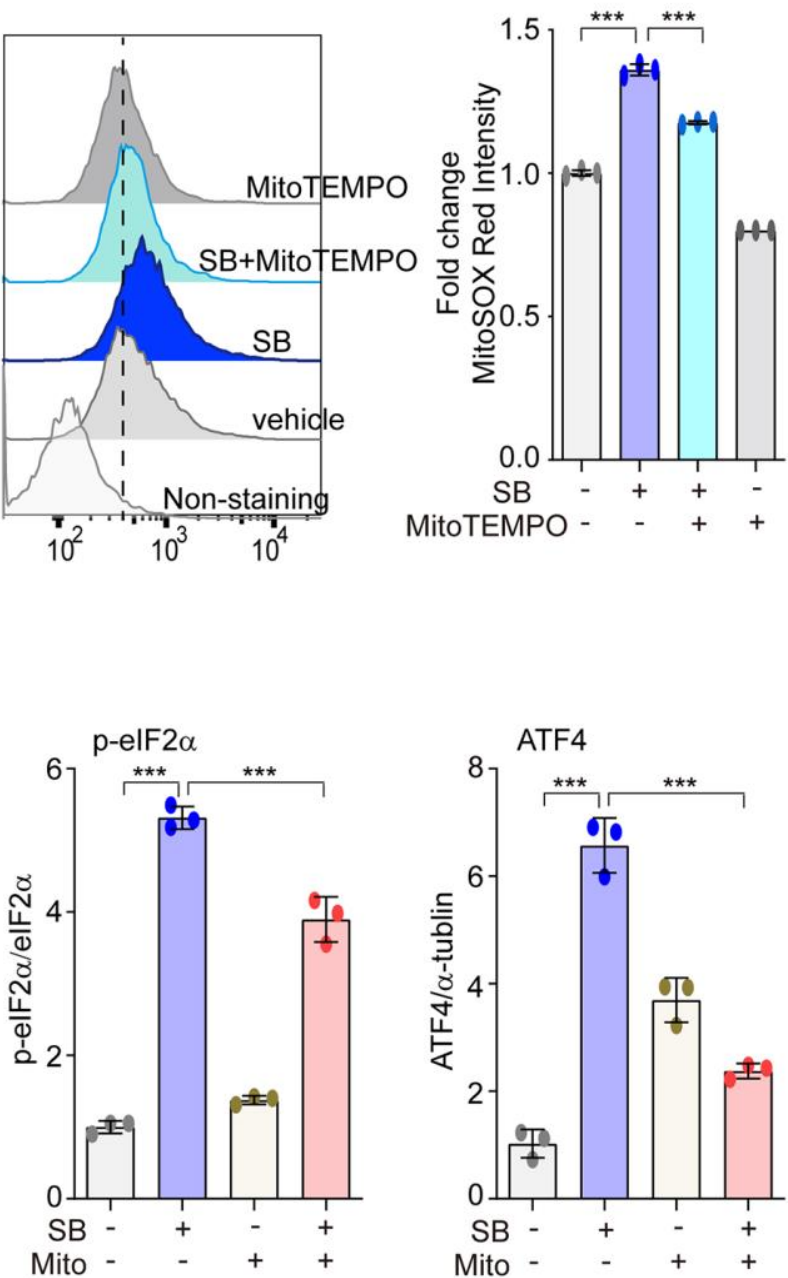

Figure 2. PERK activation with SB202190 is induced by ROS production. (A-C) SH-SY5Y cells were treated with $20 \mu \mathrm{M}$ SB202190 for 3 $\mathrm{h}$ in the presence or absence of MitoTEMPO (100 nM, $1 \mathrm{~h}$ ). (A) Cells were stained with MitoSOX (red), and then the nuclei were stained with DAPI (blue). Images were acquired by confocal microscopy. Scale bar, $10 \mu \mathrm{m}$. (B) mtROS was measured by flow cytometry. Fold changes in MitoSOX intensity are indicated at right. Data are mean \pm SD $(n=3) ;{ }^{* * *} p<0.001$. (C) Western blotting was performed to detect the phosphorylation of PERK, elF2 $\alpha$, and ATF4. 
HEK293 cells. Scavenging mtROS efficiently reduced PERK pathway activation in SB202190-treated cells (Figure 2C and Supplementary Figure 2B). These data demonstrate that SB202190 treatment is effective in activating the PERK pathway via mtROS generation, specifically in neurons.

\section{PERK is required for TFEB nuclear translocation by SB202190}

PERK is involved in translocation of TFEB into the nucleus to induce the autophagy-lysosomal pathway (ALP) [30]. In neuronal cells, the activation of ALP by an increase of TFEB nuclear translocation is critical to ameliorate amyloidogenesis. We investigated whether PERK regulates TFEB nuclear translocation in SB202190-treated SH-SY5Y cells. SB202190 induced a dose-dependent increase in TFEB nuclear translocation, as did starvation, compared to serum-fed cells (Figure 3A). TFE3 and TFEB are both members of the microphthalmia-associated transcription factor (MITF) family of the basic helix-loop-helix zipper family of transcription factors. Thus, we investigated the effects of SB202190 on TFE3 nuclear translocation. Like TFEB, the nuclear translocation of TFE3 was also increased by SB202190 (Figure 3B). In human microglial HMC3 cells, we confirmed that SB202190 enhanced TFEB nuclear translocation (Figure 3C). To investigate the mechanism for the increase of TFEB nuclear translocation by SB202190, we used a PERK inhibitor GSK2606414 or PERK knock-out MEF cells. The inhibition of PERK with GSK2606414 prevented the nuclear translocation of TFEB-GFP in response to SB202190, indicating that SB202190-regulated TFEB activity is mediated by PERK (Figure 3D). In agreement with observations in PERK inhibitor-treated cells, genetic deficiency of PERK abolished SB202190induced TFEB nuclear translocation (Figure 3E). In contrast to the requirement for PERK in SB202190induced TFEB activity; inhibition of mTOR with Torin 1 treatment promoted TFEB nuclear translocation in a manner independent of PERK. Conversely, $\mathrm{Tg}$ treatment inhibited TFEB nuclear translocation consistent with a previous report [28]. These results demonstrate that TFEB nuclear translocation is required for PERK activation in response to SB202190.

\section{The PERK-Ca ${ }^{2+}$-calcineurin pathway is required for SB202190-induced TFEB nuclear translocation}

We next investigated whether SB202190-induced PERK activation triggers $\mathrm{Ca}^{2+}$ release from the ER. In our previous study, we demonstrated that PERK activation induces the increase of cytosolic $\mathrm{Ca}^{2+}[30]$. Consistent with that finding, SB202190-induced PERK activation increased cytosolic $\mathrm{Ca}^{2+}$ in a dose-dependent manner (Figure 4A). Conversely, the PERK inhibitor GSK2606414 inhibited the cytosolic $\mathrm{Ca}^{2+}$ increase caused by SB202190 (Figure 4B). The increase of cytosolic $\mathrm{Ca}^{2+}$ in SB202190-treated cells was verified using Fluo-4AM (Figure 4C). In cells loaded with Fluo4AM in the presence of SB202190, $\mathrm{Ca}^{2+}$ levels were decreased by treatment with GSK2606414 (Figure 4C). These results suggest that the SB202190-induced increase in cytosolic $\mathrm{Ca}^{2+}$ levels is mediated via PERK activation. TFEB activity is regulated by phosphorylation [34], which retains inactive TFEB in the cytoplasm. In contrast, when dephosphorylated by the phosphatase calcineurin, TFEB translocates to the nucleus to activate transcriptional target genes [28]. We have also reported that PERK activation induced $\mathrm{Ca}^{2+}$ calcineurin-dependent TFEB regulation [30]. To explore the mechanisms of SB202190-induced TFEB nuclear translocation, SH-SY5Y cells, in the presence of SB202190, were co-incubated with the calcineurin inhibitors, FK506, or cyclosporin A (CsA). SB202190induced TFEB nuclear translocation was suppressed by FK506 (Figure 4D) and CsA (Figure 4E). These results suggest that calcineurin is partially involved in SB202190-induced TFEB nuclear translocation. We confirmed that calcineurin inhibitors block SB202190induced TFEB nuclear translocation in SH-SY5Y cells expressing TFEB-GFP (Figure 4E). These results, taken together, implicate an important role of the PERK-Ca ${ }^{2+}$ calcineurin pathway in SB202190-induced TFEB activation in human neuroblastoma cells.

\section{PERK activation by SB202190 facilitates autophagy and lysosome biogenesis via TFEB activation}

Coordination of the multiple steps in the ALP requires TFEB as a master regulator [21]. The main function of TFEB was identified in coordinating autophagy through regulating autophagosome formation and autophagosome-lysosome fusion [27]. TFEB also promotes cellular clearance through lysosomal exocytosis, a process mediated by activation of the lysosomal $\mathrm{Ca}^{2+}$ channel MCOLN1 [35]. To investigate whether SB202190-induced TFEB activation enhances the activation of the autophagy-lysosomal axis, we first measured LC3B-II and p62 levels. Consistent with a previous study [29], SB202190 increased the levels of LC3B-II and decreased p62 expression in a dosedependent manner (Figure 5A and Supplementary Figure 3A). To further investigate the effect of SB202190 on autophagy flux, we analyzed autophagosome (yellow puncta) and autolysosome (red puncta) in SH-SY5Y cells transfected with the mCherry-EGFP-LC3 reporter. Autolysosome levels were increased by SB202190 and were further enhanced following chloroquine (CQ) treatment (Figure 5B). Moreover, LC3B-II conversion in the presence of CQ 
A

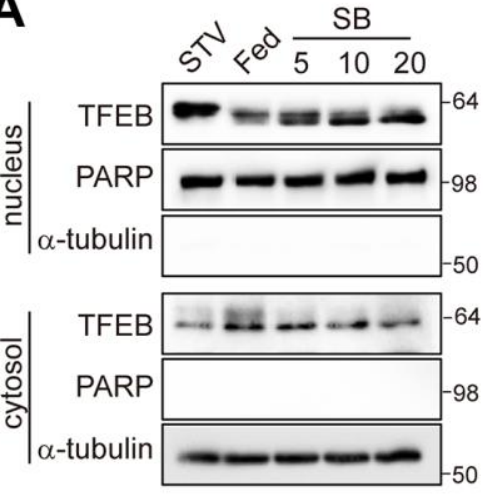

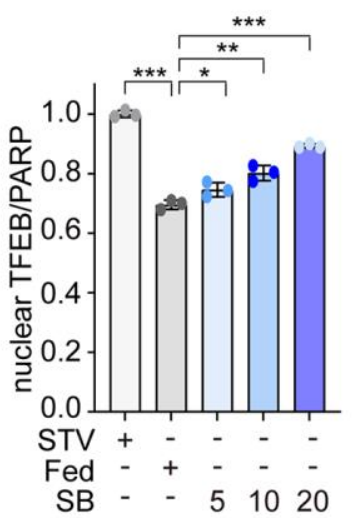
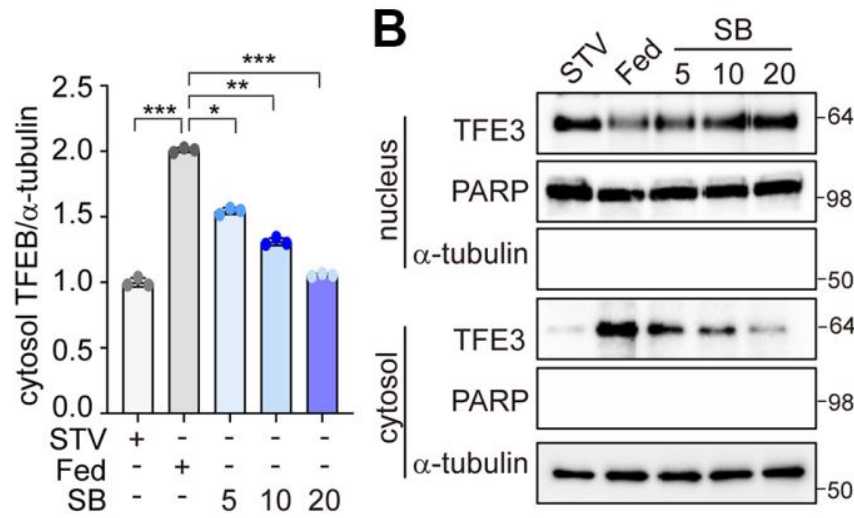

C microglial cells
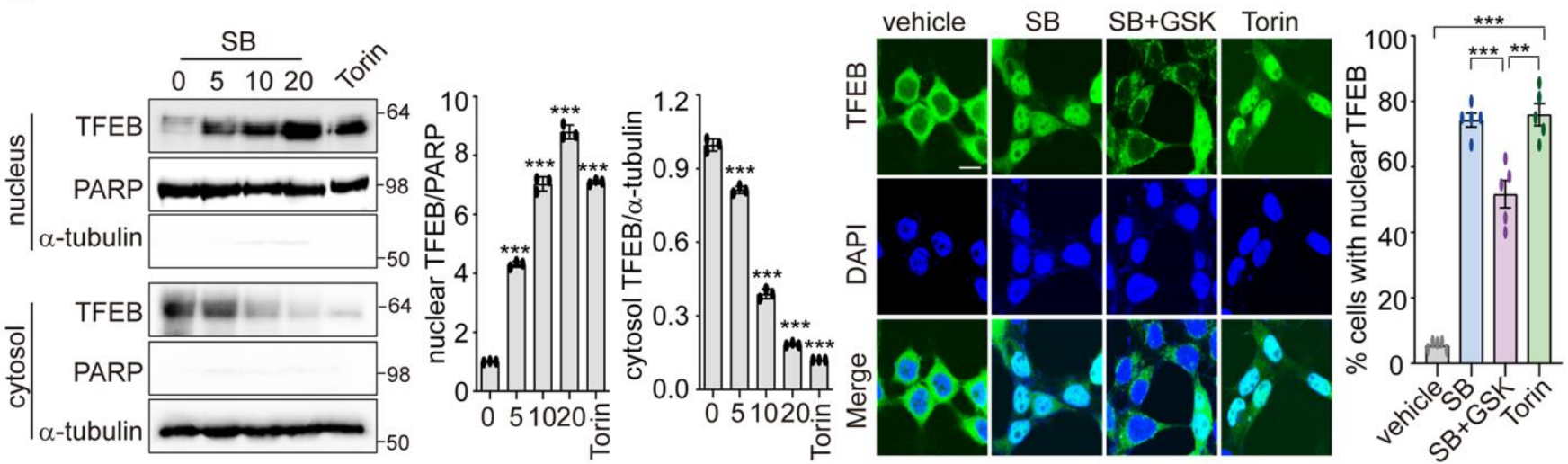

E
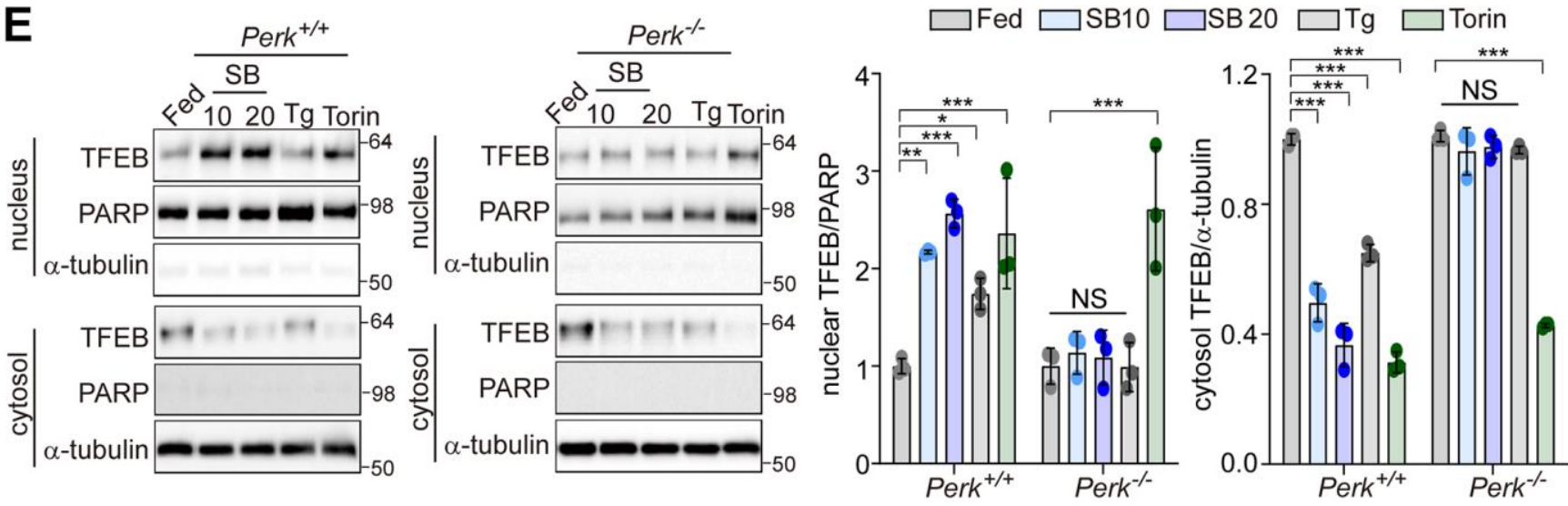

Figure 3. PERK is required for TFEB nuclear translocation by SB202190. (A, B) SH-SY5Y cells were treated with SB202190 at the indicated concentrations $(5,10$, and $20 \mu \mathrm{M})$ for $4 \mathrm{~h}$ and subjected to nuclear and cytosolic fractionation. (A) Resulting fractions were then detected with antibody against TFEB. Starvation (STV) was used as positive control. PARP and $\alpha$-tubulin were used as nuclear and cytosolic markers, respectively. Quantification of TFEB translocation is shown at the right. Data are mean $\pm \operatorname{SD}(n=3),{ }^{*} p<0.05 ; * * p<0.01 ; * * * p<0.001$. (B) The translocation of TFE3 was analyzed by western blotting. (C) The human microglial HMC3 cells were treated with SB202190 (0, 5, 10 , and 20 $\mu \mathrm{M})$ for $6 \mathrm{~h}$. Torin-1 $(2 \mu \mathrm{M})$ treatment was used as a positive control. The fractionated HMC3 cells were evaluated for TFEB translocation by western blotting (left). Quantification of TFEB translocation was analyzed (right). Data are mean \pm SD ( $n=3),{ }^{* * *} p<0.001$. (D) TFEB-EGFPtransfected SH-SY5Y cells were treated with $20 \mu \mathrm{M}$ SB202190 in the presence or absence of the PERK inhibitor (GSK2606414, GSK). Torin-1, an mTOR inhibitor, was used as a positive control. The fluorescence of TFEB was visualized by confocal microscopy (left). Scale bar: $10 \mu \mathrm{m}$. Cells were evaluated to calculate the percentage of cells showing nuclear TFEB localization. $n>20$ cells per condition (right). Data are mean \pm SD; ${ }^{* *} p<0.01$ and ${ }^{* * *} p<0.001$. (E) Perk ${ }^{+/+}$and Perk ${ }^{/-}$MEFs were treated with SB202190 (10 and $\left.20 \mu \mathrm{M}\right)$ for $6 \mathrm{~h}$ or Tg $(2 \mu \mathrm{M})$. Torin-1 $(2 \mu \mathrm{M})$ treatment was used as a positive control. Cells were detected with TFEB antibody in the nuclear and cytosol fraction by western blotting (left). Quantification of TFEB translocation was analyzed (right). Data are mean \pm SD $(n=3),{ }^{*} p<0.05 ;{ }^{* *} p<0.01 ;{ }^{* * *} p<0.001$; NS, not significant. 
was greater than that observed in SB202190-treated cells in the absence of chloroquine, suggesting that SB202190 treatment stimulates autophagic flux (Figure 5C). Next, we examined whether the effect of
SB202190 on lysosomal biogenesis is dependent on PERK activation. We observed significant and dosedependent increases in levels of lysosomal genes, including cathepsin D, cathepsin B, LAMP1, MCOLN1,
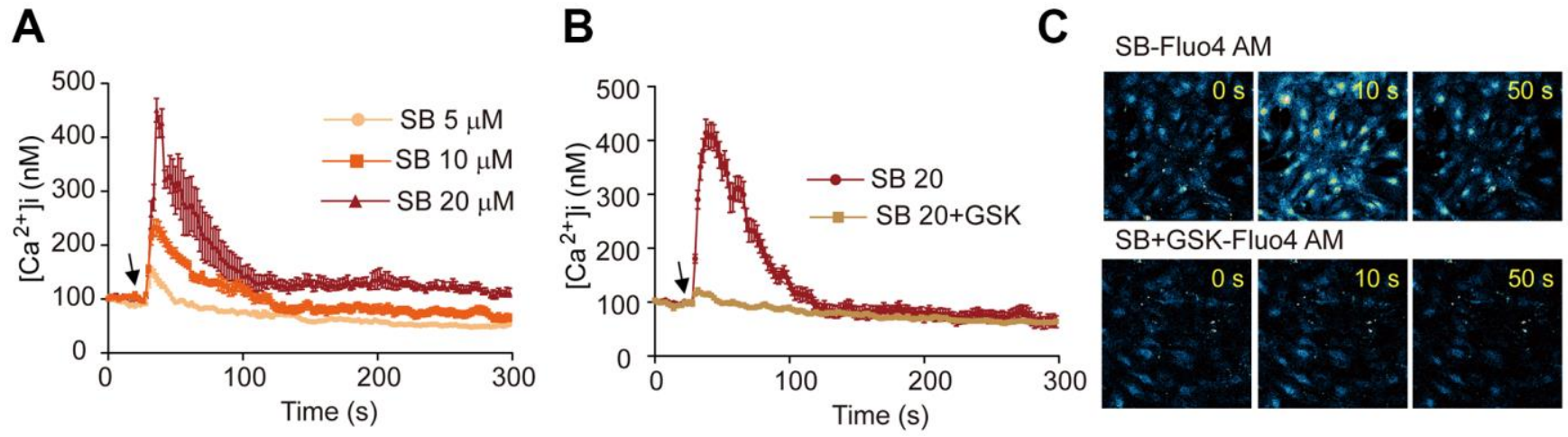

D
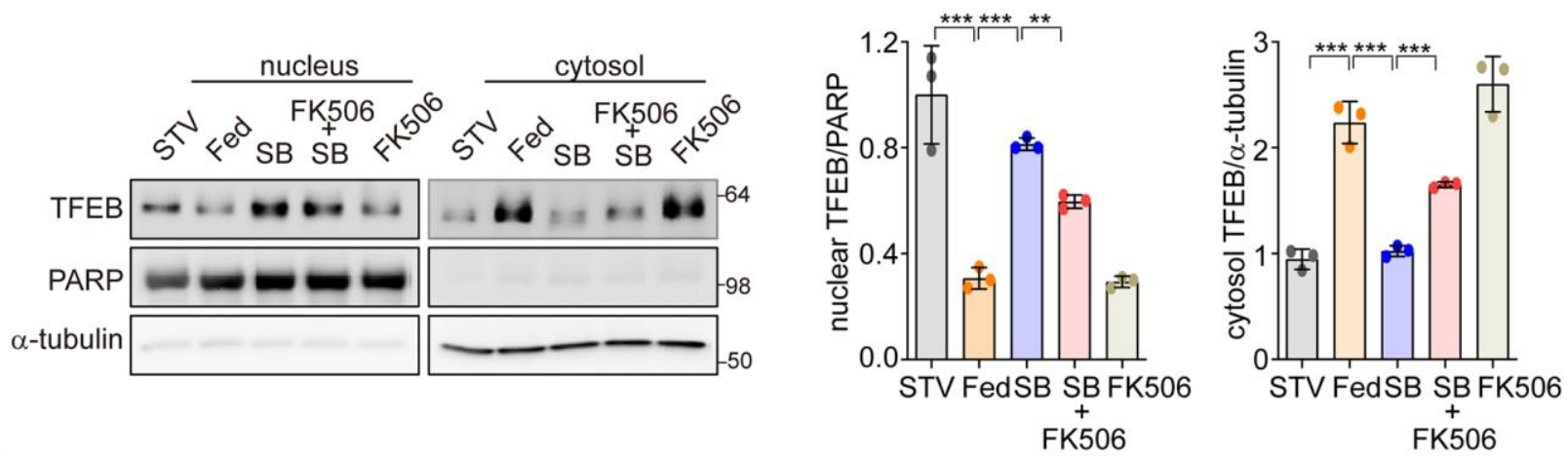

E
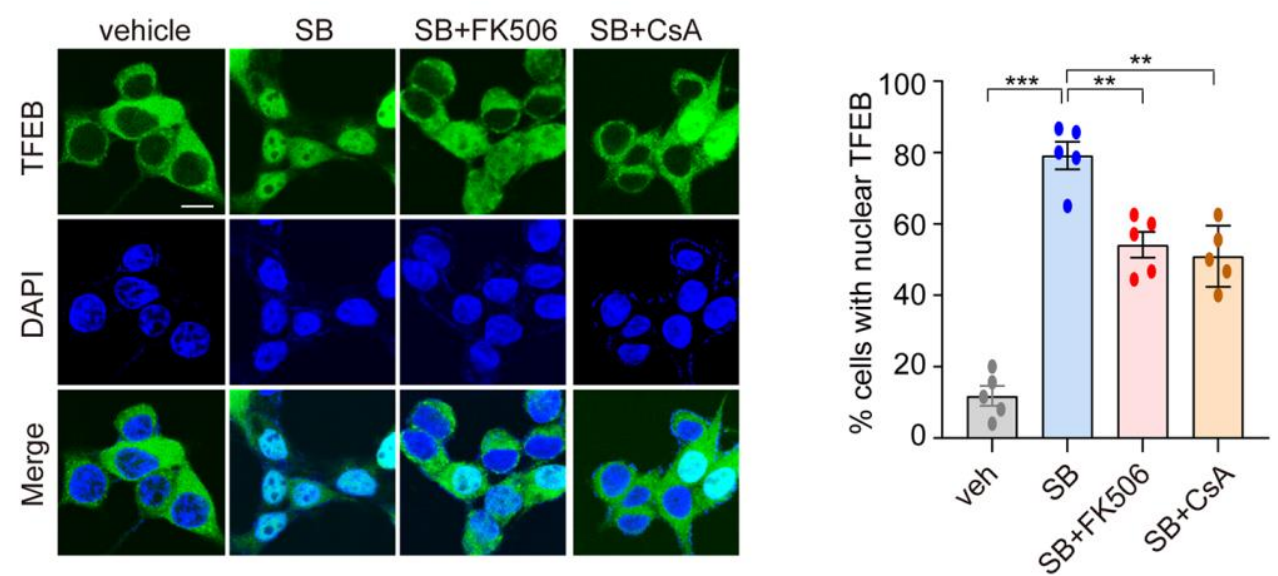

Figure 4. PERK-Ca ${ }^{2+}$-calcineurin pathway is required for SB202190-induced TFEB nuclear translocation. (A-C) The change of $\left[\mathrm{Ca}^{2+}\right]$ in MEF cells was measured using confocal microscopy after loading with Fluo-4 AM. (A) Arrow indicates the time point at which SB202190 (SB) was added. The data represent mean \pm SD from 3 independent experiments. (B, C) MEF cells were preincubated for 30 min with the PERK inhibitor GSK2606414 (1 $\mu \mathrm{M})$. Arrow indicates the time point at which $20 \mu \mathrm{M}$ SB202190 was added. The data represent mean \pm SD from 3 independent experiments. (D) SH-SY5Y cells were pretreated with FK506 (10 $\mu \mathrm{M})$ for 30 min and then treated with SB202190 (20 $\mu \mathrm{M})$ for another $3 \mathrm{~h}$. Measurement of TFEB activation was performed by western blotting of nuclear and cytoplasmic extracts. TFEB expression in the nucleus and cytoplasm was normalized to PARP and $\alpha$-tubulin, respectively (left). Quantification of TFEB translocation is shown in the right panel. Data represent mean \pm SD, $* * p<0.01 ; * * *<<0.001$. (E) TFEB-GFP-transfected SH-SY5Y cells were treated with SB202190 $(20 \mu \mathrm{M})$ for $6 \mathrm{~h}$ in the presence or absence of calcineurin inhibitors, FK506 $(10 \mu \mathrm{M})$ and Cyclosporin A (CsA, 20 $\mu \mathrm{M})$. Representative images were detected by confocal microscopy (left). Quantification of nuclear translocation of TFEB-GFP (right). $n>20$ cells per condition. Data represent mean $\pm \mathrm{SD} ; * p<0.01$ and $* * * p<0.001$. 

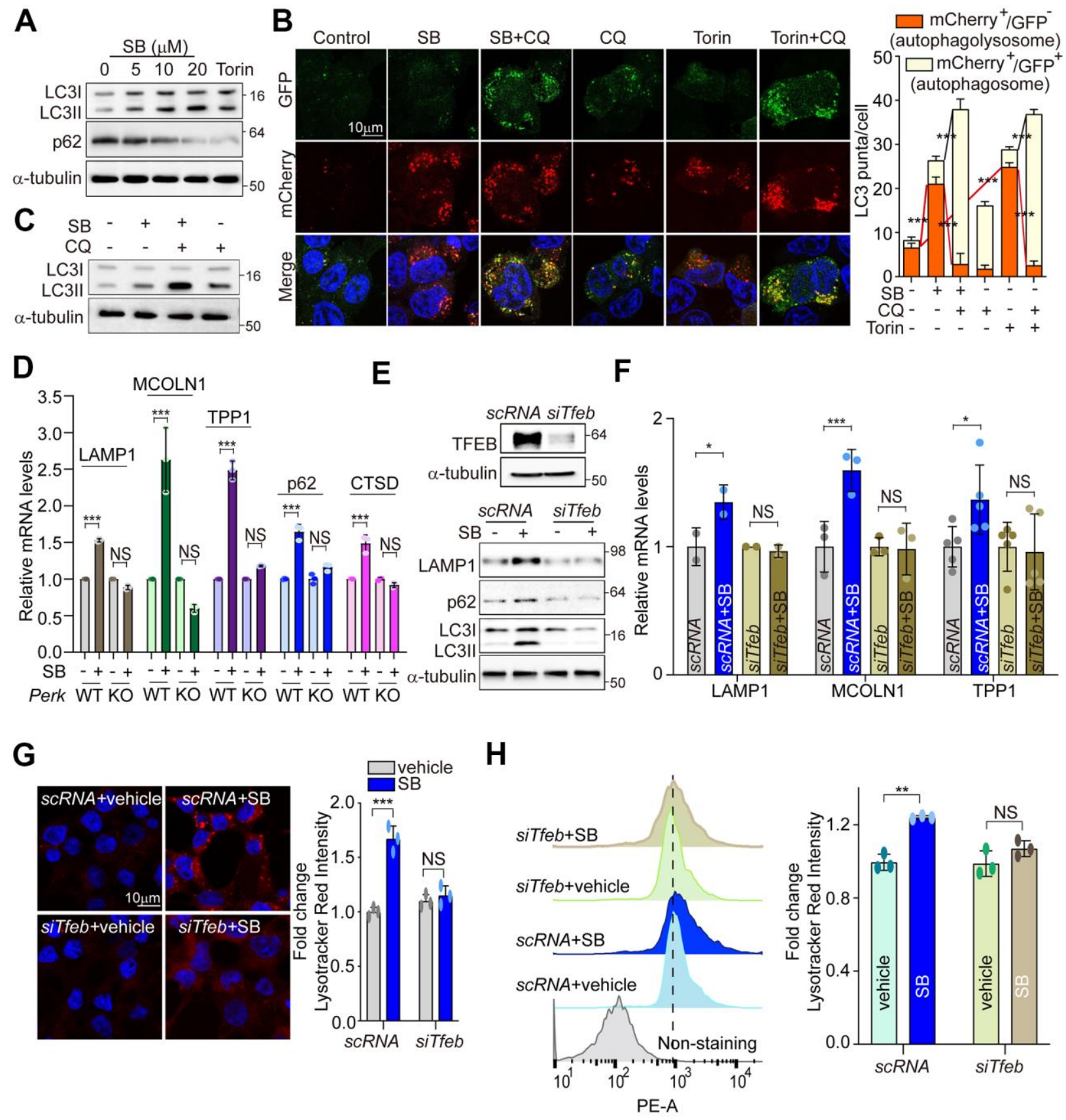

Figure 5. PERK activation by SB202190 facilitates autophagy and lysosome biogenesis via TFEB activation. (A) SH-SY5Y cells were treated for $6 \mathrm{~h}$ with SB202190 $(5,10$, and $20 \mu \mathrm{M}$ ) and subjected to western blotting by antibodies against p62 and LC3B. (B) SH-SY5Y cells were transiently transfected with mCherry-GFP-LC3 for $48 \mathrm{~h}$ and subsequently pretreated with chloroquine (CQ, $10 \mu \mathrm{M})$ for $1 \mathrm{~h}$, and then treated with SB $(20 \mu \mathrm{M})$ or Torin1 $(2 \mu \mathrm{M})$ for $6 \mathrm{~h}$. Cells were observed for fluorescence of both GFP and mCherry using confocal microscopy. The number of autolysosomes (GFP-RFP+) and autophagosomes (GFP+RFP+) per cell in each condition were quantified. Data represent mean $\pm \mathrm{SD}$; ${ }^{* * *} p<0.001$. (C) SH-SY5Y cells were treated with CQ $(10 \mu \mathrm{M})$ for $1 \mathrm{~h}$ before SB202190 $(20 \mu \mathrm{M})$ for $6 \mathrm{~h}$. The levels of LC3B-II conversion were analyzed by western blotting. (D) To check of PERK dependence in ALP-related genes, Perk $k^{+/+}$and Perk $/-$MEFs were treated with SB202190 $(20 \mu \mathrm{M})$ for $6 \mathrm{~h}$. Lysosomal genes (LAMP1, MCOLN1, TPP1, and CTSD) and autophagy gene (p62) measured by qRTPCR. Data represent mean \pm SD; $* * * p<0.001$, NS, not significant. (E-H) SH-SY5Y cells were transfected with siTfeb for $48 \mathrm{~h}$ and then treated with SB202190 $(20 \mu \mathrm{M})$ for $6 \mathrm{~h}$. (E) Cells were subjected to western blotting by using antibodies against TFEB (upper), LAMP1, p62, and LC3B (lower). (F) lysosomal genes, LAMP1, MCOLN1, and TPP1, were measured by qRT-PCR. Data are represented as mean \pm SD; ${ }^{*} p<0.05$, ${ }^{* * *} p<0.001$, NS, not significant. (G) Samples were stained with Lysotracker Red. Representative image was obtained by confocal microscopy (left). Scale bar, $10 \mu \mathrm{m}$. Quantification of lysosome intensity was determined by counting red puncta (right). Data represent mean \pm SD; ${ }^{* * *} p<0.001$, NS, not significant. (H) LysoTracker fluorescence was measured by flow cytometry. Fold changes in LysoTracker intensity are indicated at the right and are presented as mean $\pm \mathrm{SD}(\mathrm{n}=3) ;{ }^{* *} p<0.01, \mathrm{NS}$, not significant. 
and TPP1, in SB202190-treated SH-SY5Y cells (Supplementary Figure 3B, 3C). Consistent with the finding that TFEB activation in response to SB202190 treatment is dependent on PERK (Figure 3), we observed that the expression levels of ALP-related genes were increased by SB202190 treatment in erk $^{+/+}$ cells, but not in Perk $^{-/}$cells (Figure 5D and Supplementary Figure 3D). In contrast, the ability of SB202190 to increase the mRNA expression of lysosomal genes was not affected by genetic deficiency of IRE $1 \alpha$ and ATF6 $\alpha$ (Supplementary Figure 3E, 3F). In addition, deficiency of TFEB clearly suppressed SB202190-increased levels of LAMP1, p62 and LC3BII (Figure 5E, 5F and Supplementary Figure 3G). We also showed that the SB202190-dependent increase in lysosomal biogenesis required TFEB, using LysoTracker staining (Figure 5G, 5H). Overall, these results revealed that PERK activation by SB202190 regulates the ALP through TFEB activation.

\section{PERK activation by SB202190 reduces the aggregation of APP and $\alpha$-syn accumulation through TFEB-ALP activation}

PERK activation enhances the ALP via TFEB activation to degrade misfolded proteins that accumulate in neurodegenerative disorders [36, 37]. A PERK activator was shown to act as a potent agent that reduces toxicity and extends survival in murine models of HD [37]. Accumulations of APP and $\alpha$-syn have been observed in $\mathrm{AD}$ and $\mathrm{PD}$, respectively [21]. To explore whether SB202190-induced PERK activation attenuates amyloidogenesis, SH-SY5Y cells were transfected with plasmids encoding APP 695 Swedish and Indiana mutation (APPSwe/Ind) for inducing the increase of full length-APP (FL-APP), or $\alpha$-synuclein A53T mutation $(\alpha$-Syn-A53T) for inducing $\alpha$-syn aggregation. The aggregated proteins of FL-APP or $\alpha$ syn were increased by SB202190 treatment and conversely were inhibited by the PERK inhibitor, GSK2606414 (Figure 6A and Supplementary Figure 4A). In addition, SB202190 inhibited the increase of FL-APP, secreted $\mathrm{A} \beta$ and $\mathrm{CTF} \alpha / \beta$ in $\mathrm{APP}$ Swe/Ind transfected cells, which were reversed by GSK2606414 in SH-SY5Y cells (Figure 6B-6D). Consistent with the function of SB202190 in the degradation of neurotoxic proteins, LC3B-II conversion was increased after SB202190 treatment but not in the presence of the PERK inhibitor (Figure 6E and Supplementary Figure 4B), suggesting that autophagy processing may mediate the SB202190-induced decrease of APP and $\alpha$-syn. In $\mathrm{APP}^{\mathrm{S} w e / I n d}$ transfected human microglial cells, we also confirmed that the inhibition of FL-APP increase by SB202190 was recovered by GSK2606414; and that the SB202190-induced increase in LC3B-II levels was inhibited by GSK2606414 (Figure 6F). In addition, lysosomal genes increased by SB202190 were suppressed after GSK2606414 (Figure 6G). In Figure 2, we demonstrated that PERK activation by SB202910 treatment in SH-SY5Y cells was mediated by mtROS production. Thus, to confirm the role of mtROS in the degradation of aggregated mutant amyloid protein, APPSwe/Ind transfected SH-SY5Y cells were treated with MitoTEMPO. As expected, the decrease of mutant APP accumulation was reversed by MitoTEMPO treatment (Supplementary Figure 4C). As shown in Figure 5, ALP activation by SB202190 in SH-SY5Y cells was dependent on TFEB activity. Therefore, to further investigate whether the effect of SB202190 on degradation of accumulated mutant APP was dependent on TFEB, we performed co-transfection of SH-SY5Y cells with siTfeb and APPSwe/Ind. TFEB deficient cells failed to degrade FL-APP after SB202190 treatment, indicating that TFEB activation by SB202190 is essential to activate ALP for the reduction of neurotoxic proteins in SH-SY5Y cells (Figure 6H, 6I). Taken together, our results demonstrate that SB202190induced ALP activation which is responsible for degradation of mutant APP and $\alpha$-syn accumulation requires PERK-dependent TFEB activation.

\section{DISCUSSION}

This study identifies a novel effect of SB202190 in promoting PERK activation, independent of p38 MAPK inhibition, in the cellular response to mitigate amyloidogenesis in human neuroblastoma cells. We demonstrate that SB202190-induced PERK activation activates TFEB nuclear translocation, leading to induction of the ALP. The ALP is involved in the degradation of misfolded proteins that accumulate in many neurodegenerative diseases such as $\mathrm{AD}, \mathrm{PD}$, and HD [21]. Thus, enhancing the activity of the ALP in neurodegenerative diseases has been extensively studied as a strategy for therapeutic intervention [19]. Interestingly, only SB202190, among several other p38 MAPK inhibitors, can enhance TFEB-mediated ALP activation [29]. SB202190 triggers the release of $\mathrm{Ca}^{2+}$ from the $\mathrm{ER}$ and subsequent calcineurin activation, leading to TFEB-induced ALP activation, apart from its known function as a p38 MAPK inhibitor [29]. Our data also show that SB202190 promotes TFEB nuclear translocation in SH-SY5Y cells. We further found that SB202190 specifically induces the activation of PERK among the three known ER sensors, PERK, IRE1 $\alpha$ and ATF6. In animal models of neurodegenerative disease and in human post-mortem tissue from many neurodegenerative diseases $[7,38]$, the PERK pathway has been associated with disease progression. Genetic and pharmacological modulation of the PERK pathway is the focus of new therapeutic strategies of neurodegenerative diseases $[7,39,40]$. Therefore, our 

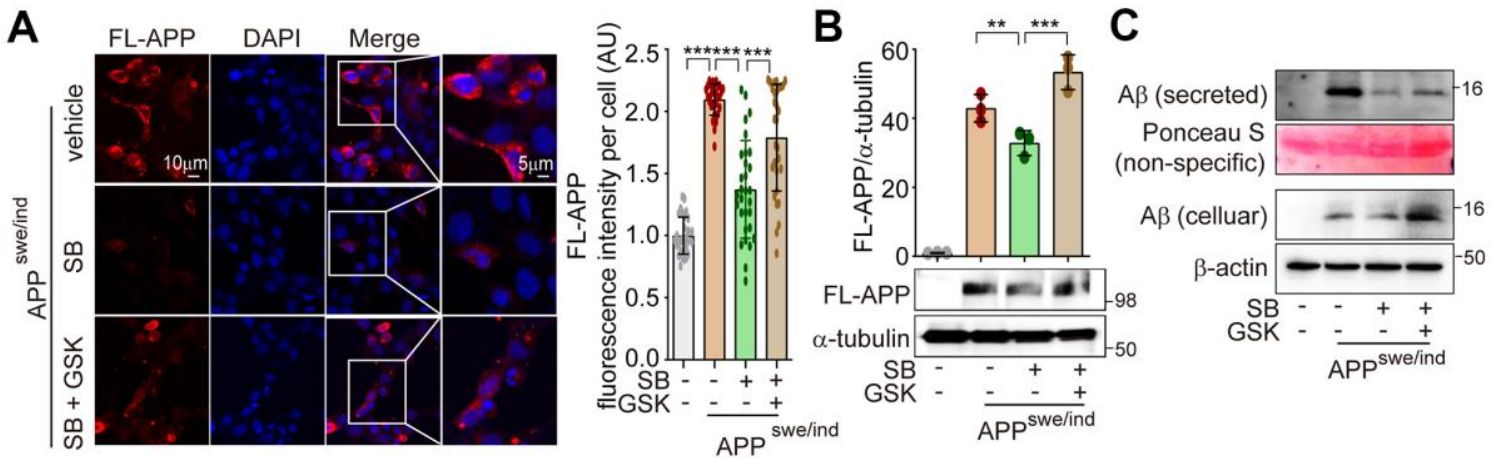

D

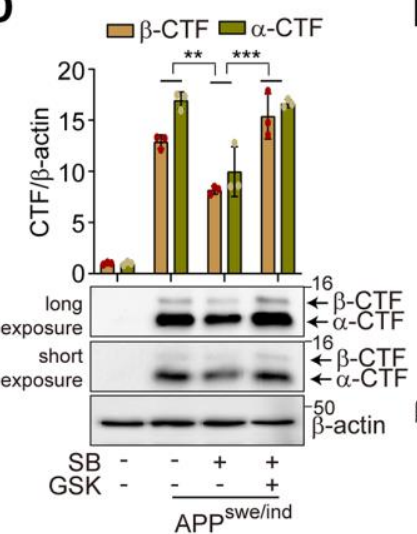

E

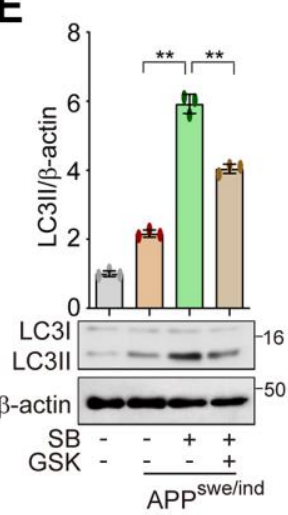

$\mathbf{F}$

Microglial cells

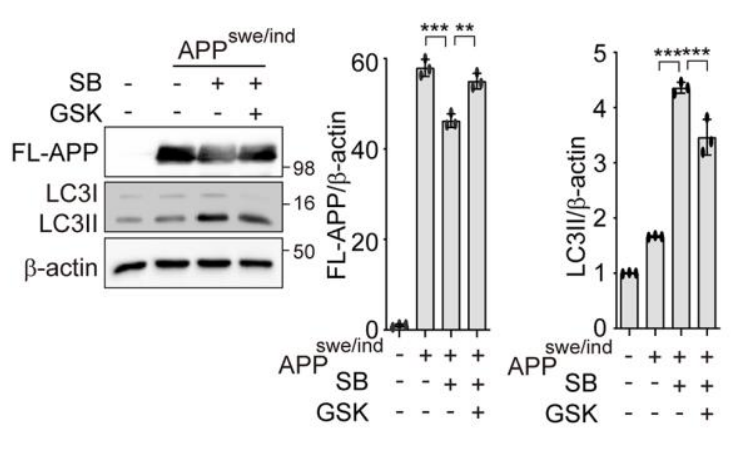

$\mathbf{G}$

Microglial cells

H

I

I $\frac{\text { APP }^{\text {swe/ind }}}{\frac{\text { SCRNA }}{\frac{\text { sitfeb }}{\text { vehicle SB }}}}$
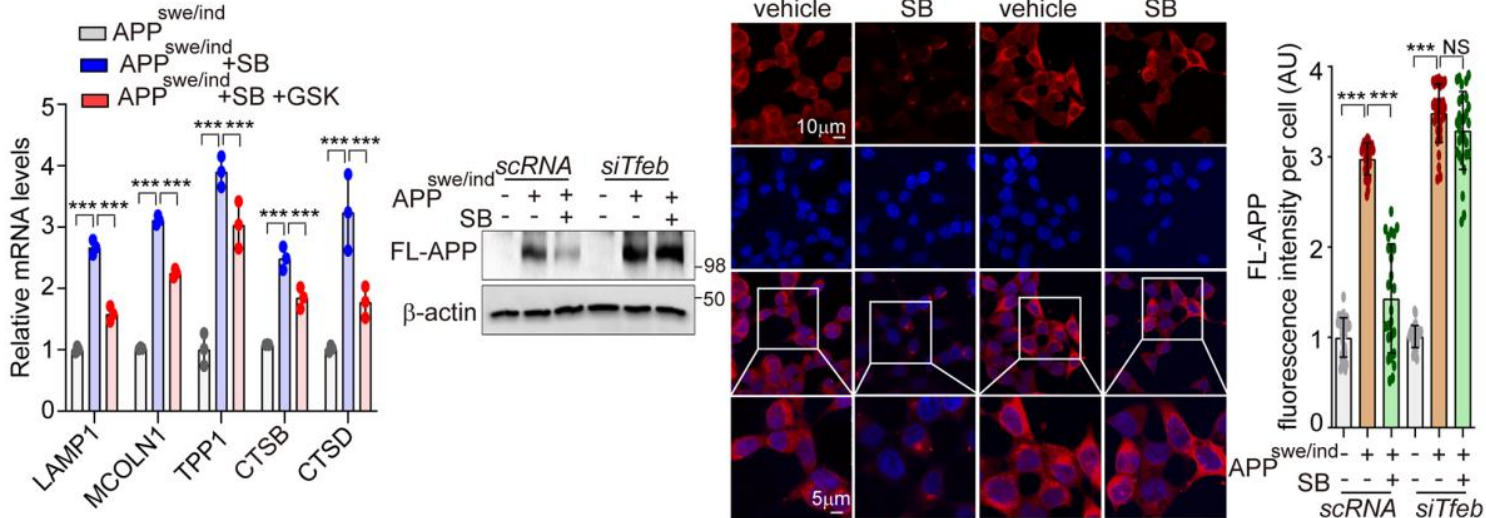

Figure 6. PERK activation by SB202190 reduces the aggregation of APP accumulation through TFEB-ALP activation in SH-SY5Y cells. (A, B) SH-SY5Y cells were transfected with pCAX-APP-Swe/Ind (APPswe/ind) for $48 \mathrm{~h}$. Under this condition, cells were treated with the PERK inhibitor, GSK2606414 (1 $\mu \mathrm{M}, 1 \mathrm{~h})$, before SB202190 (20 $\mu \mathrm{M}, 12 \mathrm{~h})$ treatment. (A) Cells were stained with DAPI and immunostained with anti-FL-APP antibody. Representative image of FL-APP was observed by confocal microscopy (left) and quantification of FL-APP intensity (right). Data represent mean \pm SD; ${ }^{* * *} p<0.001$. (B) Western blotting was performed to detect the expression levels of FL-APP. Quantification of FL-APP expression was shown in the upper panel. (C) The levels of intracellular $A \beta$ and secreted $A \beta$ were detected by western blotting. (D, E) The cells were subjected to western blotting by antibodies against $\alpha / \beta-C T F$ (D) and LC3B (E). Quantification of $\alpha / \beta-C T F$ and LC3B-II conversion was analyzed (upper). Data are mean \pm SD $(n=3), * * p<0.01 ; * * * p<0.001$. (F, G) HMC3 cells were transfected with pCAX-APPSwe/Ind (APPswe/ind) for $48 \mathrm{~h}$ and then treated with SB202190 (20 $\mu \mathrm{M}, 12 \mathrm{~h})$ in the presence or absence of PERK inhibitor, GSK2606414 (1 $\mu \mathrm{M}$, $1 \mathrm{~h}$ ). (F) Cells were detected to antibodies against APP and LC3B by western blotting. Quantification of FL-APP and LC3B-II conversion was analyzed. Data are mean \pm SD $(n=3),{ }^{* *} p<0.01 ; * * *<<0.001$. (G) Lysosomal genes, LAMP1, MCOLN1, TPP1, CTSB, and CTSD were measured by qRT-PCR. Data are represented as mean \pm SD, $* * * p<0.001$. (H, I) SH-SY5Y cells were co-transfected with pCAX-APP-Swe/Ind (APPswe/ind) and siTfeb for $48 \mathrm{~h}$ and were treated with SB202190 (20 $\mu \mathrm{M}, 12 \mathrm{~h})$. (H) Cells were detected to antibody against FL-APP by western blotting. (I) Cells were stained with antibody against FL-APP and DAPI. Representative image of FL-APP was obtained by confocal microscopy (left) and quantification of FL-APP intensity (right). Data represent as mean \pm SD; ${ }^{* * *} p<0.001$ and not significant (NS). 
findings for SB202190-induced PERK activation in neuroblastoma cells may provide insight into strategies to resolve pathological amyloidogenesis.

Our results also indicate that PERK activation by SB202190 is mediated by mtROS, resulting in the increase of cytosolic $\mathrm{Ca}^{2+}$ and the activation of calcineurin. In our previous studies, we found that a mild increase of mtROS levels leads to PERK activation, $\mathrm{Ca}^{2+}$ release from the ER, and calcineurindependent TFEB activation in hepatocytes [30, 41]. Moreover, calcineurin plays a detrimental role in damaged neurons. Notably, the cytoprotective effects of calcineurin in acute brain injuries are PERK-dependent [18]. Thus, our study suggests that the protective role of PERK-calcineurin in neurons is due to an increase TFEB nuclear translocation. Calcineurin dephosphorylates TFEB, leading to increased autophagy induction and lysosomal biogenesis [28]. We demonstrate that SB202190 increases autophagy-related genes and autophagolysosome formation. In addition, our data show that SB202190-induced ALP activation is dependent on PERK but not IRE1 $\alpha$ and ATF6 in human neuroblastoma cells. To verify the amelioration of amyloidogenesis via SB202190-induced ALP activation, SH-SY5Y cells were transfected with mutant FL-APP or $\alpha$-syn as in vitro models of AD or PD, respectively [31, 36, 42]. Accumulation of either FLAPP or $\alpha$-syn in SH-SY5Y cells was mitigated with SB202190 treatment. In particular, the decrease of amyloidogenesis with SB202190 required PERK activation. Thus, we also demonstrated that SB202190induced PERK activation ameliorates amyloidogenesis in $\mathrm{AD}$ or PD models via mtROS production using the mitochondria-targeting antioxidant MitoTEMPO.

In summary, our data reveal a novel cytoprotective role for SB202190 as a PERK activator in human neuroblastoma cells. Our findings also suggest that SB202190 activates PERK via an increase of mtROS, leading to calcineurin activation. Subsequently, PERK activation by SB202190 leads to an increase in TFEB nuclear translocation through calcineurin-dependent dephosphorylation of TFEB. Finally, SB202190-PERKTFEB activation attenuates amyloidogenesis via the increase of the ALP (see schema in Figure 7). We anticipate that SB202190 and related compounds targeting PERK can be developed as therapeutics to ameliorate amyloidogenesis in neurodegenerative disorders.

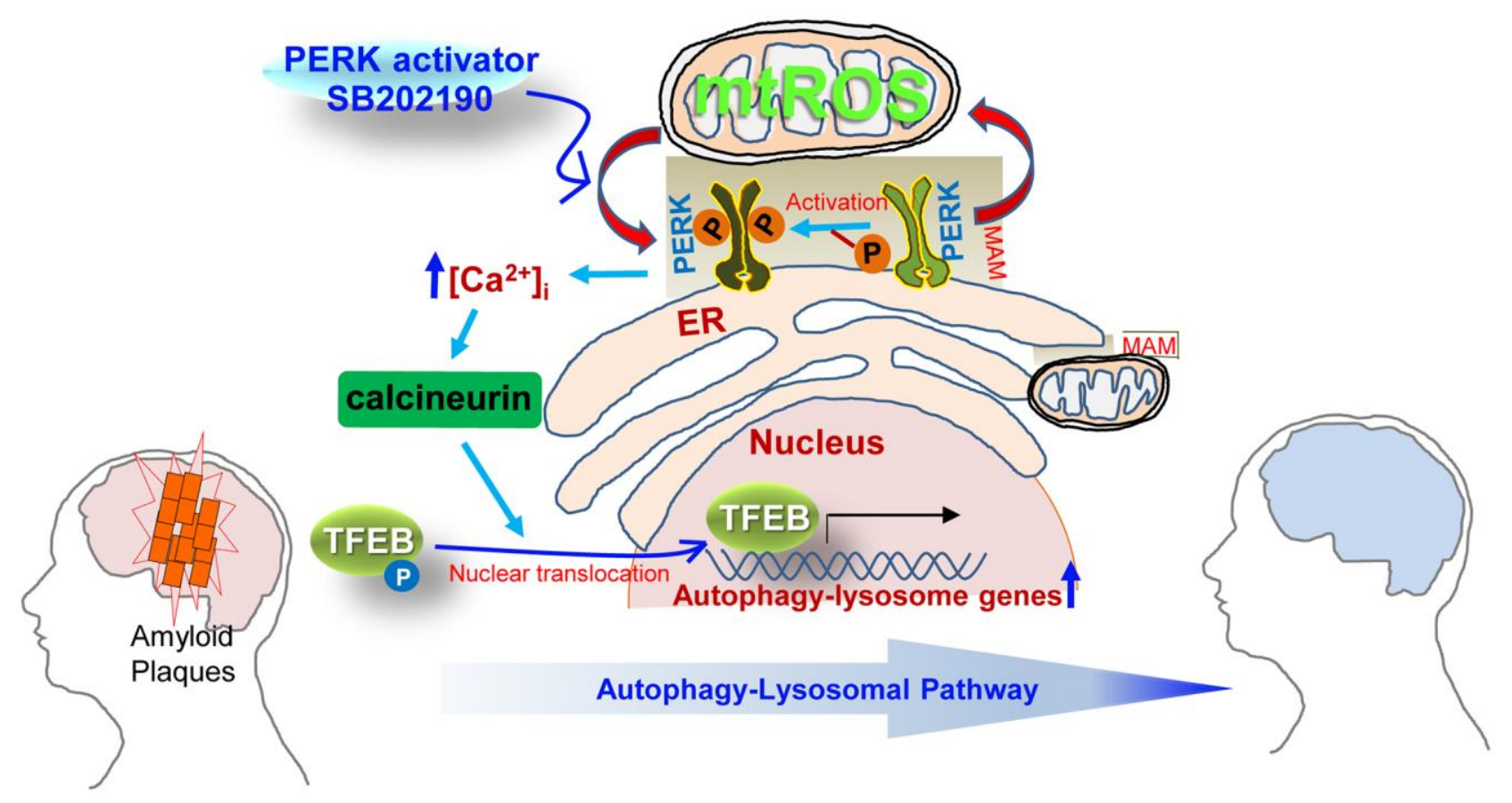

Figure 7. Schematic overview of the mechanisms by which SB202190 ameliorates amyloidogenesis via PERK activation. PERK, a tethering molecule of the mitochondrial-associated ER membrane (MAM), is activated by mitochondrial ROS (mtROS) in response to treatment with the PERK activator (SB202190). Activated PERK leads to increase in cytosolic Ca ${ }^{2+}$ levels and subsequently promotes the translocation of TEFB into the nucleus via the calcineurin-dependent dephosphorylation TFEB, which culminates in the increased transcription of autophagy-lysosome related genes. The increase of autophagy-lysosomal pathway (ALP) by PERK activation enhances the degradation of misfolded proteins that accumulate in neurodegenerative disorders. Therefore, the PERK-TFEB-ALP pathway activated by SB202190 suggests the novel target for ameliorating amyloidogenesis. 


\section{MATERIALS AND METHODS}

\section{Reagents}

SB202190, GSK2606414, thapsigargin (Tg), FK506, chloroquine (CQ), and cyclosporin A (CsA) were purchased from Sigma-Aldrich (St Louis, MO, USA). Torin 1 was from (Tocris-Biotechne, Minneapolis, MN, USA).

\section{Cell lines}

The human neuroblastoma cell line SH-SY5Y, and the human embryonic kidney cell line HEK293 were grown in Dulbecco's Modified Eagle medium, DMEM (Gibco, Grand Island, NY, USA), supplemented with $10 \%$ fetal bovine serum (Gibco, Melbourne, Australia) and 1\% penicillin-streptomycin solution (Gibco, Grand Island, NY, USA). HMC3 human microglial cell line was cultured in MEM, with 10\% fetal bovine serum (Gibco, Melbourne, Australia) and 1\% penicillin-streptomycin solution (Gibco, Grand Island, NY, USA). Perk ${ }^{+/+}$and Perk $^{-/}$mouse embryonic fibroblasts (MEFs) were cultured in DMEM supplemented with 1\% MEM nonessential amino acid (Gibco). Ire $1 \alpha^{+/+}, \operatorname{Irel} \alpha^{-/}$, Atf $6 \alpha^{+/+}$, and Atf6 $\alpha^{-/-}$mouse hepatocytes, which were kindly provided by Dr. S. H. Back (University of Ulsan, Ulsan, Korea), were maintained in 199 medium, with $1 \%$ MEM non-essential amino acid solution. Cells were grown in humidified incubators, at $37^{\circ} \mathrm{C}$ with $5 \% \mathrm{CO}_{2}$.

\section{Western blot}

Total protein extracts from harvested-cells were prepared in RIPA buffer (Thermo Scientific, Waltham, MA, USA) containing phosphatase and protease inhibitors (Sigma-Aldrich), and the protein concentration was determined using the BCA protein assay kit (Pierce Biotechnology, Rockford, IL, USA) using bovine serum albumin (BSA) as standard. Aliquots of protein were boiled at $95^{\circ} \mathrm{C}$ in $2 \mathrm{X}$ Lamelli buffer (Bio-Rad, Hercules, CA, USA) for $5 \mathrm{~min}$. Proteins were separated by SDS-PAGE and transferred to polyvinylidene difluoride membrane (Millipore, Burlington, MA, USA). The membrane was blocked with 5\% nonfat milk (BD bioscience, San Jose, CA, USA) in phosphate buffered saline-Tween 20 (PBS-T), and then the membrane was immunoblotted with primary antibodies as follows: p-PERK (1:1000, Signalway Antibody, Baltimore, MD, USA), PERK (1:1000, Cell Signaling, Danvers, MA, USA), p-eIF2 $\alpha$ (1:1000, Cell Signaling), eIF2 $\alpha$ (1:1000, Cell Signaling), ATF4 (1:1000, Cell Signaling), TFEB (1:1000, Bethyl Laboratories, Montgomery, TX, USA), PARP (1:2000, Cell Signaling), LC3B (1:2000, Novus Biologicals, Centennial, CO, USA), p62 (1:10000), LAMP1 (1:1000, Abcam, Cambridge, MA, USA), anti- amyloid precursor protein, C-Terminal (1:2000, SigmaAldrich), $\alpha$-synuclein (1:2000, Cell Signaling), TFE3 (1:1000, Sigma-Aldrich), $\beta$-amyloid, 1-16 (1:1000, BioLegend), $\alpha$-tubulin (1:2000, Cell Signaling), and $\beta$ actin (1:2500, Thermo Scientific). This was followed by incubation in HRP-conjugated secondary antibodies. Immunoblots were detected using an ECL substrate (Pierce Biotechnology) and analyzed using an Azure Biosystems C300 analyzer (Azure Biosystems, Dublin, CA, USA).

\section{Measurement of mitochondrial ROS}

SH-SY5Y and HEK293 cells were treated with SB202190 $(20 \mu \mathrm{M})$ for $3 \mathrm{~h}$ in the presence or absence of $100 \mathrm{nM}$ Mito-TEMPO (Sigma-Aldrich) for $1 \mathrm{~h}$. After treatment, cells were stained with $5 \mu \mathrm{M}$ MitoSOX Red (Invitrogen, Carlsbad, CA, USA) at $37^{\circ} \mathrm{C}$ for $30 \mathrm{~min}$. For flow cytometry, cells were trypsinized and then washed three times with $1 \mathrm{X}$ PBS. mtROS were detected by using a FACSCanto flow cytometry system (BD Bioscience, CA, USA). Fluorescence data were analyzed with FlowJo software (C). For confocal microscope, cells were fixed with formalin solution for $30 \mathrm{~min}$ and then washed three times with $1 \mathrm{X}$ PBS. The cells were stained DAPI (Invitrogen). Samples were visualized by using an Olympus FV1200 confocal microscope (Olympus, Tokyo, Japan).

\section{RT-PCR and qRT-PCR}

Total RNA was isolated from cells using by QIAzol Lysis reagent (QIAGEN, Valencia, CA, USA). Reverse transcription of $2 \mu \mathrm{g}$ RNA was performed using M-MLV reverse transcriptase (Promega, Madison, WI, USA). The PCR-based amplification was done using synthesizedcDNA. The following primers were human GAPDH (fccacccatggcaaattccatggca, r-tctagacggcaggtcaggtccacc), human Xbp1 (f-ccttgtagttgagaaccagg, r-ggggcttggtatat atgtgg), and human GRP78 (f-cgaggaggaggacaagaagg, rttgtttgcccacctccaata). To analyze real-time quantitative PCR (RT-qPCR), the synthesized cDNA was amplified with SYBR Green qPCR Master Mix on an ABI 7500 Fast Real-Time PCR System (Applied Biosystems, CA, USA), The following qRT-PCR primers were human GAPDH (f-caatgacccettcatcctc, r-agcatcgccccacttgatt), human CTSB (f-agtggagaatggcacacccta, r-aagagccattgtcac ccca), human CTSD (f-cttcgacaacctgatgcagc, r-tacttggagt ctgtgccacc), human LAMP1 (f-cgtacctttccaacagcagc, rcgctcacgttgtacttgtcc), human MCOLN1 (f-gagtgg gtgcgacaagtttc, r-tgttctcttcceggaatgtc), and TPP1 (f-gatccc agctctcctcaatac, r- gccatttttgcaccgtgtg), human TPP1 (f-ga tcccagctctcctcaatac, r- gccattttgcaccgtgtg), mouse GAPDH (f-cggcctcaccccatttg, r-gggaagcccatcaccatct), mouse MCOLN1 (f-gcgcctatgacaccatcaa, r-tatcctggactg ctcgat), mouse TPP1 (f-aagccaggcctacatactcaga, r-ccaagt 
gcttcctgcagtttaga), mouse LAMP1 (f-taatggccagcttc tctgcctcctt, r-aggctggggtcagaaacattttctt), mouse CatD (faactgctggacatcgcttgct, r-cattcttcacgtaggtgctgga), and mouse p62 (f-gaggcaccccgaaacatgg, r-acttatagcg agttcccacca).

\section{Transfection}

pEGFP-N1-TFEB, pBABE-puro mCherry-EGFPLC3B, $\alpha$-Syn-A53T, and pCAX-APP-Swe/Ind were purchased from Addgene (Watertown, MA, USA) and were transfected into cells via Lipofectamine ${ }^{\mathrm{TM}} 2000$ (Invitrogen) in accordance with the manufacturer's protocol. To knockdown the genes of p38, Perk and Tfeb, SH-SY5Y cells were transfected with scramble siRNA (scRNA) (Ambion, Austin, TX, USA), as the control siRNA, p38 (Cell Signaling), Perk and Tfeb siRNA (Santa Cruz Biotechnology, CA, USA) using Lipofectamine ${ }^{\mathrm{TM}} 2000$ (Invitrogen) method according to the manufacturer's protocol. After $36 \mathrm{~h}$, cells were treated with indicated drugs.

\section{Fluorescence cell imaging}

For imaging of TFEB-GFP and mCherry-GFP-LC3, SH-SY5Y cells grown on coverslips were transfected with plasmids for $36 \mathrm{~h}$ and then were treated with indicated drugs. After treatment, cells were fixed with $10 \%$ formalin solution (Sigma-Aldrich) for $20 \mathrm{~min}$ at RT, followed by three times washes and DAPI staining. After additional three washes, the slides were mounted with mounting medium (Sigma-Aldrich). Cells were visualized using the Olympus FV1200 confocal microscope (Olympus).

\section{Immunofluorescence}

To detect the FL-APP and $\alpha$-synuclein using confocal microscope, SH-SY5Ys were incubated on coverslips and transfected with pCAX-APP695-Swe/Ind and $\alpha$ syn-A53T, followed by the administration of SB202190, Mito-TEMPO, or PERK inhibitor. After treatment, the samples were fixed with $10 \%$ neutralbuffered formalin solution (Sigma Aldrich) for $20 \mathrm{~min}$. The cells were subsequently permeabilized with $0.1 \%$ Triton X-100 and then blocked with 3\% BSA for 30 $\min$ at RT. The cells were stained with anti-FL-APP (1:500, Sigma-Aldrich) or anti- $\alpha$-synuclein (1:500, Cell Signaling) antibodies for overnight at $4^{\circ} \mathrm{C}$. Alexa-Fluor 594 anti-rabbit (1:500, Invitrogen) secondary antibody was added for $2 \mathrm{~h}$, at RT. Then, the cells were washed with $1 \mathrm{X}$ PBS-T followed by DAPI staining. Images of the cells were obtained using an Olympus FV1200 confocal microscope (Olympus). The intensity of FL-APP and $\alpha$-syn was analyzed by using ImageJ software.

\section{Subcellular fractionation}

Nuclear and cytosolic fractions were prepared by using nuclear/cytosol fractionation kit (Biovision, Milpitas, CA, USA). Cells were harvested, and subcellular fractionation was performed according to the manufacturer's instructions. Briefly, cell pellets were resuspended with cytosolic extraction buffer A (CEBA) and CEB-B. After $10 \mathrm{~min}$, the lysates were centrifuged at $4^{\circ} \mathrm{C}$ for $5 \mathrm{~min}$ at $16,000 \mathrm{X} \mathrm{g}$ in a microcentrifuge to obtain cytosolic protein containing supernatant. The remaining pellets were resuspended in nuclear extraction buffer (NEB) and vortexed for $15 \mathrm{~s}$. This step was repeated every $10 \mathrm{~min}$, for five times. Samples were centrifuged at $4^{\circ} \mathrm{C}$ for $10 \mathrm{~min}$ at 16,000 $\mathrm{X} g$ to acquire nuclear extraction. The purity of cytoplasmic and nuclear fractions was subjected to western blotting by anti- $\beta$-actin and PARP antibodies.

\section{Measurement of intracellular $\mathrm{Ca}^{2+}$ concentration}

MEF cells were plated and cultured on confocal dishes. The cells were loaded with $5 \mu \mathrm{M}$ fluo-4 AM (Invitrogen) in Hanks' balanced salt solution (HBSS) with $1 \%$ bovine serum albumin at $37^{\circ} \mathrm{C}$ for $40 \mathrm{~min}$ as described previously [30]. The cells were washed three times with HBSS. Changes of intracellular $\mathrm{Ca}^{2+}$ concentration $\left(\left[\mathrm{Ca}^{2+}\right]_{\mathrm{i}}\right)$ were determined at $488 \mathrm{~nm}$ excitation/530 nm emission using an air-cooled argon laser system. The emitted fluorescence at $530 \mathrm{~nm}$ was collected using a photomultiplier. The image was scanned using a confocal microscope (Nikon, Japan). For the calculation of $\left[\mathrm{Ca}^{2+}\right]_{i}$, the method of Tsien et al. [43] was used with the following equation: $\left[\mathrm{Ca}^{2+}\right]_{\mathrm{i}}=$ $\mathrm{Kd}(\mathrm{F}-\mathrm{Fmin}) /(\mathrm{Fmax}-\mathrm{F})$, where $\mathrm{Kd}$ is $345 \mathrm{nM}$ for fluo-4 and $\mathrm{F}$ is the observed fluorescence level. Each tracing was calibrated for the maximal intensity (Fmax) by addition of ionomycin $(8 \mu \mathrm{M})$ and for the minimal intensity (Fmin) by addition of EGTA $(50 \mathrm{mM})$ at the end of each measurement.

\section{Lysosome quantification}

The lysosomal contents were assessed using LysoTracker Red (Invitrogen) following manufacturer's instructions. After treatment, cells in fresh medium were stained with LysoTracker Red for $1 \mathrm{~h}$ followed by extensive washing and then transferred into tubes for quantification of lysosome fluorescence using a FACSCanto flow cytometry system (BD Bioscience). Data were analyzed by FlowJo software (Tree Star). For measurement of lysosome intensity using confocal image, the stained cells were fixed with $10 \%$ formalin solution followed by DAPI staining. Samples expressing fluorescence were identified by Olympus FV1200 confocal microscope. 


\section{Statistical analysis}

Data were analyzed with Prism (GraphPad Software, San Diego, CA, USA). All values are presented as mean \pm SD. Statistical analyses were performed using Student's $t$-test or one-way ANOVA with Tukey post hoc tests.

\section{Abbreviations}

PERK: protein kinase $\mathrm{R}$ (PKR)-like endoplasmic reticulum (ER) kinase; UPR: unfolded protein response; ALP: autophagy-lysosome pathway; TFEB: transcription factor-EB; IRE1 $\alpha$ : inositol-requiring transmembrane kinase/endoribonuclease-1 $\alpha$; ATF6: activating transcription factor 6; AD: Alzheimer's diseases; PD: Parkinson's diseases; HD: Huntington's diseases; $\mathrm{A} \beta$ : amyloid $\beta$; ER: endoplasmic reticulum; $\alpha$-syn: $\alpha$-synuclein; mtROS: mitochondrial ROS; MAMs: mitochondria-associated ER membranes; Tg: thapsigargin; CsA: cyclosporin A; CQ: chloroquine.

\section{AUTHOR CONTRIBUTIONS}

Conceptualization: H.T.C., Y.J.; Data curation: J.P., Y.J.; Formal analysis: M.D., J.P., Y.C., S.-Y.R., T.H.T.N., J.H.G.; Investigation: S.-A J., B.-S.K., Y. R., J.W.P., U.-H.K.,; Project administration: Y.J., H.T.C.; Resources: J.W.P., U.-H.K., Y.J., H.T.C.; Writing, review and editing: M.D, J.P., B.-S.K.,Y.-J. S., J.W.P., S.W.R., Y.J, H.T.C.

\section{CONFLICTS OF INTEREST}

The authors declare that they have no conflicts of interest.

\section{FUNDING}

This work was supported by the Priority Research Centers Program through the National Research Foundation of Korea (NRF) funded by the Ministry of Education (2014R1A6A1030318, NRF-2020R1A2C1009192) to H.T.C., NRF-2020R1A2C1006470 to Y. J, NRF2021R1F1A1052424 to R. Y.

\section{REFERENCES}

1. Knowles TP, Vendruscolo M, Dobson CM. The amyloid state and its association with protein misfolding diseases. Nat Rev Mol Cell Biol. 2014; 15:384-96. https://doi.org/10.1038/nrm3810 PMID:24854788

2. Sipe JD. Amyloidosis. Annu Rev Biochem. 1992; 61:947-75.

https://doi.org/10.1146/annurev.bi.61.070192.004503 PMID: 1497327
3. Chiti F, Dobson CM. Protein misfolding, functional amyloid, and human disease. Annu Rev Biochem. 2006; 75:333-66.

https://doi.org/10.1146/annurev.biochem.75.101304. 123901 PMID: 16756495

4. Ron D, Walter P. Signal integration in the endoplasmic reticulum unfolded protein response. Nat Rev Mol Cell Biol. 2007; 8:519-29. https://doi.org/10.1038/nrm2199 PMID:17565364

5. Rozpedek W, Markiewicz L, Diehl JA, Pytel D, Majsterek I. Unfolded Protein Response and PERK Kinase as a New Therapeutic Target in the Pathogenesis of Alzheimer's Disease. Curr Med Chem. 2015; 22:3169-84. https://doi.org/10.2174/092986732266615081810425 4 PMID:26282939

6. Bell MC, Meier SE, Ingram AL, Abisambra JF. PERKopathies: An Endoplasmic Reticulum Stress Mechanism Underlying Neurodegeneration. Curr Alzheimer Res. 2016; 13:150-63.

https://doi.org/10.2174/1567205013666151218145 $\underline{431}$ PMID:26679859

7. Hughes D, Mallucci GR. The unfolded protein response in neurodegenerative disorders - therapeutic modulation of the PERK pathway. FEBS J. 2019; 286:342-55.

https://doi.org/10.1111/febs.14422 PMID:29476642

8. Harding HP, Zhang Y, Ron D. Protein translation and folding are coupled by an endoplasmic-reticulumresident kinase. Nature. 1999; 397:271-4. https://doi.org/10.1038/16729 PMID:9930704

9. Cox JS, Shamu CE, Walter P. Transcriptional induction of genes encoding endoplasmic reticulum resident proteins requires a transmembrane protein kinase. Cell. 1993; 73:1197-206.

https://doi.org/10.1016/0092-8674(93)90648-A PMID: 8513503

10. Wang Y, Shen J, Arenzana N, Tirasophon W, Kaufman RJ, Prywes R. Activation of ATF6 and an ATF6 DNA binding site by the endoplasmic reticulum stress response. J Biol Chem. 2000; 275:27013-20. https://doi.org/10.1016/S0021-9258(19)61473-0 PMID: 10856300

11. Devi L, Ohno M. PERK mediates elF2 $\alpha$ phosphorylation responsible for BACE1 elevation, CREB dysfunction and neurodegeneration in a mouse model of Alzheimer's disease. Neurobiol Aging. 2014; 35:2272-81. https://doi.org/10.1016/i.neurobiolaging.2014.04.031 PMID:24889041

12. Ma T, Trinh MA, Wexler AJ, Bourbon C, Gatti E, Pierre P, Cavener DR, Klann E. Suppression of elF $2 \alpha$ kinases alleviates Alzheimer's disease-related plasticity and memory deficits. Nat Neurosci. 2013; 16:1299-305. 
https://doi.org/10.1038/nn.3486

PMID:23933749

13. Colla E, Coune P, Liu Y, Pletnikova O, Troncoso JC, Iwatsubo T, Schneider BL, Lee MK. Endoplasmic reticulum stress is important for the manifestations of a-synucleinopathy in vivo. J Neurosci. 2012; 32:3306-20.

https://doi.org/10.1523/JNEUROSCI.5367-11.2012 PMID:22399753

14. Hayashi T, Rizzuto R, Hajnoczky G, Su TP. MAM: more than just a housekeeper. Trends Cell Biol. 2009; 19:81-8.

https://doi.org/10.1016/j.tcb.2008.12.002 PMID:19144519

15. Verfaillie T, Rubio N, Garg AD, Bultynck G, Rizzuto R, Decuypere JP, Piette J, Linehan C, Gupta S, Samali A, Agostinis P. PERK is required at the ER-mitochondrial contact sites to convey apoptosis after ROS-based ER stress. Cell Death Differ. 2012; 19:1880-91. https://doi.org/10.1038/cdd.2012.74 PMID:22705852

16. Zhang Y, Sun R, Geng S, Shan Y, Li X, Fang W. Porcine Circovirus Type 2 Induces ORF3-Independent Mitochondrial Apoptosis via PERK Activation and Elevation of Cytosolic Calcium. J Virol. 2019; 93:e01784-18.

https://doi.org/10.1128/JVI.01784-18 PMID:30651358

17. Bollo $M$, Paredes RM, Holstein D, Zheleznova $N$, Camacho $\mathrm{P}$, Lechleiter JD. Calcineurin interacts with PERK and dephosphorylates calnexin to relieve ER stress in mammals and frogs. PLoS One. 2010; 5:e11925.

https://doi.org/10.1371/journal.pone.0011925 PMID:20700529

18. Chen $\mathrm{Y}$, Holstein DM, Aime S, Bollo M, Lechleiter JD. Calcineurin $\beta$ protects brain after injury by activating the unfolded protein response. Neurobiol Dis. 2016; 94:139-56.

https://doi.org/10.1016/i.nbd.2016.06.011

PMID:27334877

19. Menzies FM, Fleming A, Rubinsztein DC. Compromised autophagy and neurodegenerative diseases. Nat Rev Neurosci. 2015; 16:345-57.

https://doi.org/10.1038/nrn3961 PMID:25991442

20. Yamamoto A, Simonsen A. The elimination of accumulated and aggregated proteins: a role for aggrephagy in neurodegeneration. Neurobiol Dis. 2011; 43:17-28. https://doi.org/10.1016/j.nbd.2010.08.015 PMID:20732422

21. Martini-Stoica $H, X u Y$, Ballabio A, Zheng $H$. The Autophagy-Lysosomal Pathway in Neurodegeneration: A TFEB Perspective. Trends Neurosci. 2016; 39:221-34. https://doi.org/10.1016/i.tins.2016.02.002 PMID:26968346

22. Tsunemi T, Ashe TD, Morrison BE, Soriano KR, Au J, Roque RA, Lazarowski ER, Damian VA, Masliah E, La Spada AR. PGC-1 $\alpha$ rescues Huntington's disease proteotoxicity by preventing oxidative stress and promoting TFEB function. Sci Transl Med. 2012; 4:142ra97.

https://doi.org/10.1126/scitranslmed.3003799 PMID:22786682

23. Decressac $M$, Mattsson $B$, Weikop $P$, Lundblad $M$, Jakobsson J, Björklund A. TFEB-mediated autophagy rescues midbrain dopamine neurons from $\alpha$-synuclein toxicity. Proc Natl Acad Sci USA. 2013; 110:E1817-26. https://doi.org/10.1073/pnas.1305623110 PMID:23610405

24. Xiao Q, Yan P, Ma X, Liu H, Perez R, Zhu A, Gonzales E, Tripoli DL, Czerniewski L, Ballabio A, Cirrito JR, Diwan A, Lee JM. Neuronal-Targeted TFEB Accelerates Lysosomal Degradation of APP, Reducing $A \beta$ Generation and Amyloid Plaque Pathogenesis. J Neurosci. 2015; 35:12137-51.

https://doi.org/10.1523/JNEUROSCI.0705-15.2015 PMID:26338325

25. Rehli M, Den Elzen N, Cassady Al, Ostrowski MC, Hume DA. Cloning and characterization of the murine genes for bHLH-ZIP transcription factors TFEC and TFEB reveal a common gene organization for all MiT subfamily members. Genomics. 1999; 56:111-20.

https://doi.org/10.1006/geno.1998.5588 PMID:10036191

26. Sardiello $M$, Palmieri $M$, di Ronza $A$, Medina DL, Valenza M, Gennarino VA, Di Malta C, Donaudy F, Embrione V, Polishchuk RS, Banfi S, Parenti G, Cattaneo $E$, Ballabio A. A gene network regulating lysosomal biogenesis and function. Science. 2009; 325:473-7.

https://doi.org/10.1126/science.1174447 PMID:19556463

27. Settembre C, Di Malta C, Polito VA, Garcia Arencibia M, Vetrini F, Erdin S, Erdin SU, Huynh T, Medina D, Colella $P$, Sardiello M, Rubinsztein DC, Ballabio A. TFEB links autophagy to lysosomal biogenesis. Science. 2011; 332:1429-33.

https://doi.org/10.1126/science.1204592 PMID:21617040

28. Medina DL, Di Paola S, Peluso I, Armani A, De Stefani D, Venditti R, Montefusco S, Scotto-Rosato A, Prezioso C, Forrester A, Settembre C, Wang W, Gao Q, et al. Lysosomal calcium signalling regulates autophagy through calcineurin and TFEB. Nat Cell Biol. 2015; 17:288-99.

https://doi.org/10.1038/ncb3114

PMID:25720963 
29. Yang C, Zhu Z, Tong BC, Iyaswamy A, Xie WJ, Zhu Y, Sreenivasmurthy SG, Senthilkumar K, Cheung KH, Song JX, Zhang HJ, Li M. A stress response p38 MAP kinase inhibitor SB202190 promoted TFEB/TFE3-dependent autophagy and lysosomal biogenesis independent of p38. Redox Biol. 2020; 32:101445.

https://doi.org/10.1016/i.redox.2020.101445

PMID:32037305

30. Kim HJ, Joe Y, Rah SY, Kim SK, Park SU, Park J, Kim J, Ryu J, Cho GJ, Surh YJ, Ryter SW, Kim UH, Chung HT. Carbon monoxide-induced TFEB nuclear translocation enhances mitophagy/mitochondrial biogenesis in hepatocytes and ameliorates inflammatory liver injury. Cell Death Dis. 2018; 9:1060.

https://doi.org/10.1038/s41419-018-1112-x

PMID:30333475

31. Chartier-Harlin MC, Kachergus J, Roumier C, Mouroux V, Douay X, Lincoln S, Levecque C, Larvor L, Andrieux J, Hulihan M, Waucquier N, Defebvre L, Amouyel P, et al. Alpha-synuclein locus duplication as a cause of familial Parkinson's disease. Lancet. 2004; 364:1167-9.

https://doi.org/10.1016/S0140-6736(04)17103-1 PMID:15451224

32. Joe Y, Kim S, Kim HJ, Park J, Chen Y, Park HJ, Jekal SJ, Ryter SW, Kim UH, Chung HT. FGF21 induced by carbon monoxide mediates metabolic homeostasis via the PERK/ATF4 pathway. FASEB J. 2018; 32:2630-43. https://doi.org/10.1096/fj.201700709RR PMID:29295856

33. Chen Y, Joe Y, Park J, Song HC, Kim UH, Chung HT. Carbon monoxide induces the assembly of stress granule through the integrated stress response. Biochem Biophys Res Commun. 2019; 512:289-94.

https://doi.org/10.1016/j.bbrc.2019.03.017

PMID:30885431

34. Settembre C, Zoncu R, Medina DL, Vetrini F, Erdin S, Erdin S, Huynh T, Ferron M, Karsenty G, Vellard MC, Facchinetti V, Sabatini DM, Ballabio A. A lysosome-tonucleus signalling mechanism senses and regulates the lysosome via mTOR and TFEB. EMBO J. 2012; 31:1095-108.

https://doi.org/10.1038/emboj.2012.32

PMID:22343943

35. Medina DL, Fraldi A, Bouche V, Annunziata F, Mansueto G, Spampanato C, Puri C, Pignata A, Martina JA, Sardiello M, Palmieri M, Polishchuk R, Puertollano $\mathrm{R}$, Ballabio A. Transcriptional activation of lysosomal exocytosis promotes cellular clearance. Dev Cell. 2011; 21:421-30.

https://doi.org/10.1016/i.devcel.2011.07.016

PMID:21889421
36. Song HC, Chen Y, Chen Y, Park J, Zheng M, Surh YJ, Kim UH, Park JW, Yu R, Chung HT, Joe Y. GSK-3 $\beta$ inhibition by curcumin mitigates amyloidogenesis via TFEB activation and anti-oxidative activity in human neuroblastoma cells. Free Radic Res. 2020; 54:918-30. https://doi.org/10.1080/10715762.2020.1791843 PMID:32623920

37. Ganz J, Shacham T, Kramer M, Shenkman M, Eiger $H$, Weinberg N, lancovici O, Roy S, Simhaev L, Da'adoosh $B$, Engel $\mathrm{H}$, Perets $\mathrm{N}$, Barhum $\mathrm{Y}$, et al. A novel specific PERK activator reduces toxicity and extends survival in Huntington's disease models. Sci Rep. 2020; 10:6875. https://doi.org/10.1038/s41598-020-63899-4 PMID: $\underline{32327686}$

38. Moreno JA, Radford $H$, Peretti $D$, Steinert JR, Verity $N$, Martin MG, Halliday M, Morgan J, Dinsdale D, Ortori CA, Barrett DA, Tsaytler P, Bertolotti A, et al. Sustained translational repression by elF $2 \alpha-P$ mediates prion neurodegeneration. Nature. 2012; 485:507-11.

https://doi.org/10.1038/nature11058 PMID:22622579

39. Radford $H$, Moreno JA, Verity $N$, Halliday $M$, Mallucci GR. PERK inhibition prevents tau-mediated neurodegeneration in a mouse model of frontotemporal dementia. Acta Neuropathol. 2015; 130:633-42.

https://doi.org/10.1007/s00401-015-1487-z PMID:26450683

40. Imran M, Mahmood S. An overview of human prion diseases. Virol J. 2011; 8:559.

https://doi.org/10.1186/1743-422X-8-559

PMID:22196171

41. Kim HJ, Joe Y, Kim SK, Park SU, Park J, Chen Y, Kim J, Ryu J, Cho GJ, Surh YJ, Ryter SW, Kim UH, Chung HT. Carbon monoxide protects against hepatic steatosis in mice by inducing sestrin-2 via the PERK-elF2 $\alpha$-ATF4 pathway. Free Radic Biol Med. 2017; 110:81-91. https://doi.org/10.1016/j.freeradbiomed.2017.05.026 PMID:28578014

42. Zhang $X$, Yin $W K$, Shi $X D$, Li $Y$. Curcumin activates Wnt/ $\beta$-catenin signaling pathway through inhibiting the activity of GSK-3 $\beta$ in APPswe transfected SY5Y cells. Eur J Pharm Sci. 2011; 42:540-6. https://doi.org/10.1016/i.ejps.2011.02.009 PMID:21352912

43. Tsien RY, Pozzan T, Rink TJ. T-cell mitogens cause early changes in cytoplasmic free $\mathrm{Ca}+$ and membrane potential in lymphocytes. Nature. 1982; 295:68-71. https://doi.org/10.1038/295068a0 PMID: $\underline{6799829}$ 


\section{SUPPLEMENTARY MATERIALS}

\section{Supplementary Figures}
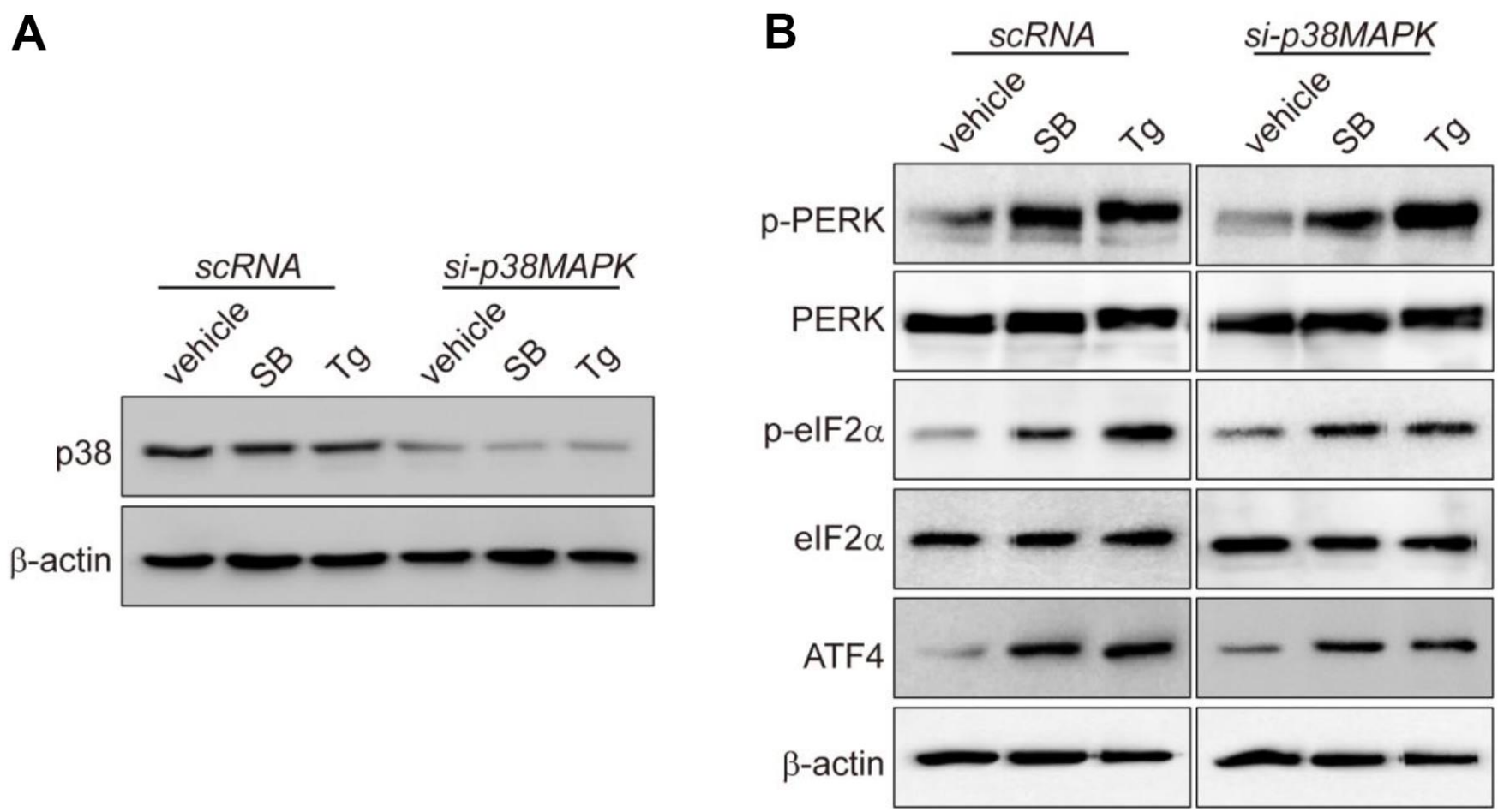

Supplementary Figure 1. PERK activation with SB202190 is independent of p38 MAPK-related to Figure 1. (A, B) SH-SY5Y cells were transfected with control siRNA (scRNA) or si-p38 MAPK for $48 \mathrm{~h}$ and then treated with $20 \mu \mathrm{M} \mathrm{SB202190} \mathrm{for} 6 \mathrm{~h}$ or $1 \mu \mathrm{M} \mathrm{Tg}$ for $30 \mathrm{~min}$. Cell lysates were evaluated for p38 MAPK knockdown by western blot (A) and detected with antibodies against p-PERK, PERK, p-elF2 $\alpha$, elF2 $\alpha$, and ATF4 by western blotting (B). 
A

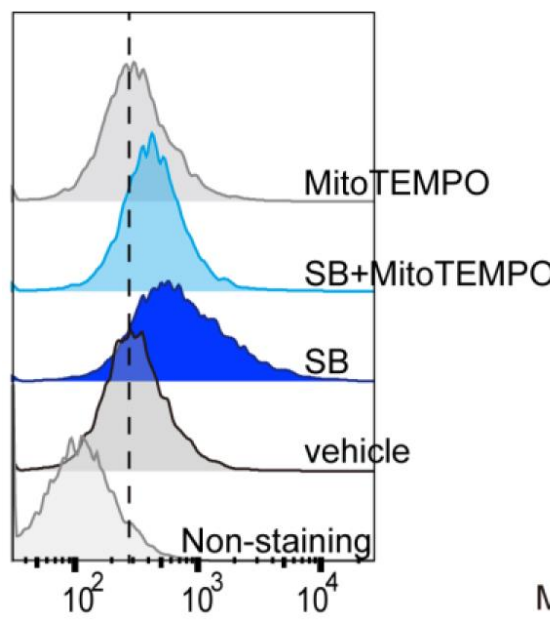

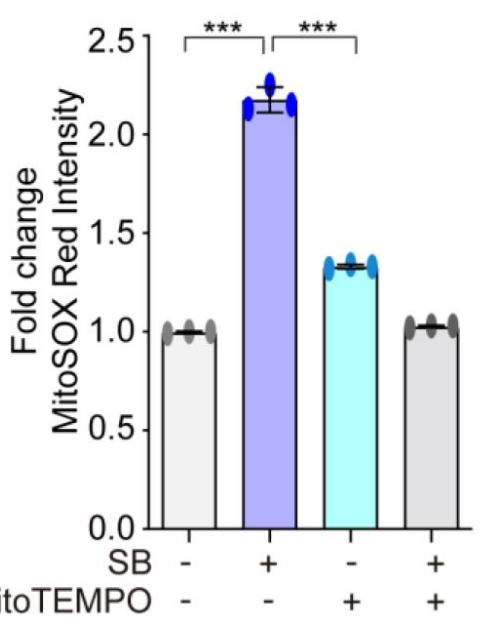

B

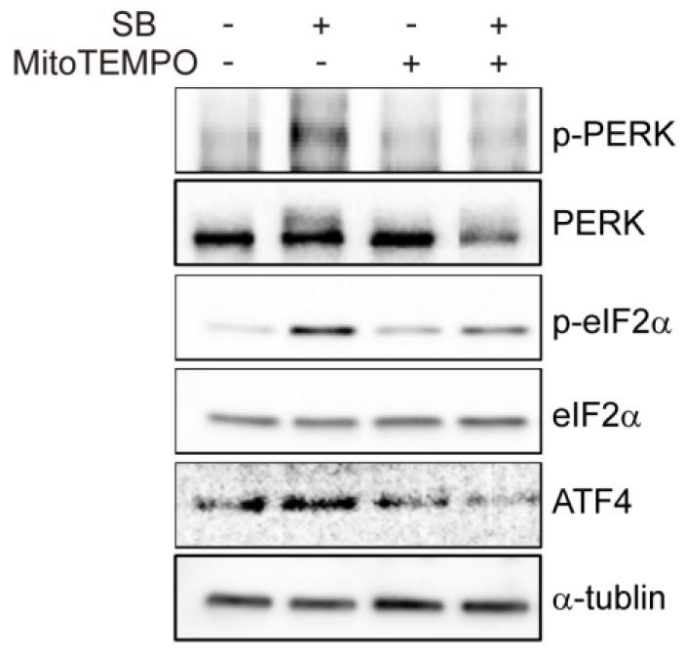

Supplementary Figure 2. PERK activation with SB202190 is induced by ROS production- related to Figure 2. (A, B) HEK293 cells were pretreated with MitoTEMPO (100 nM) for $1 \mathrm{~h}$ and then treated with $20 \mu \mathrm{M} \mathrm{SB202190} \mathrm{for} 3 \mathrm{~h}$. (A) MitoSOX fluorescence was analyzed by flow cytometry (left), and quantification of fluorescence was normalized (right). Data are mean \pm SD $(n=3), * * * p<0.001$. (B) The activation of PERK was analyzed by western blotting using the indicated antibodies. 


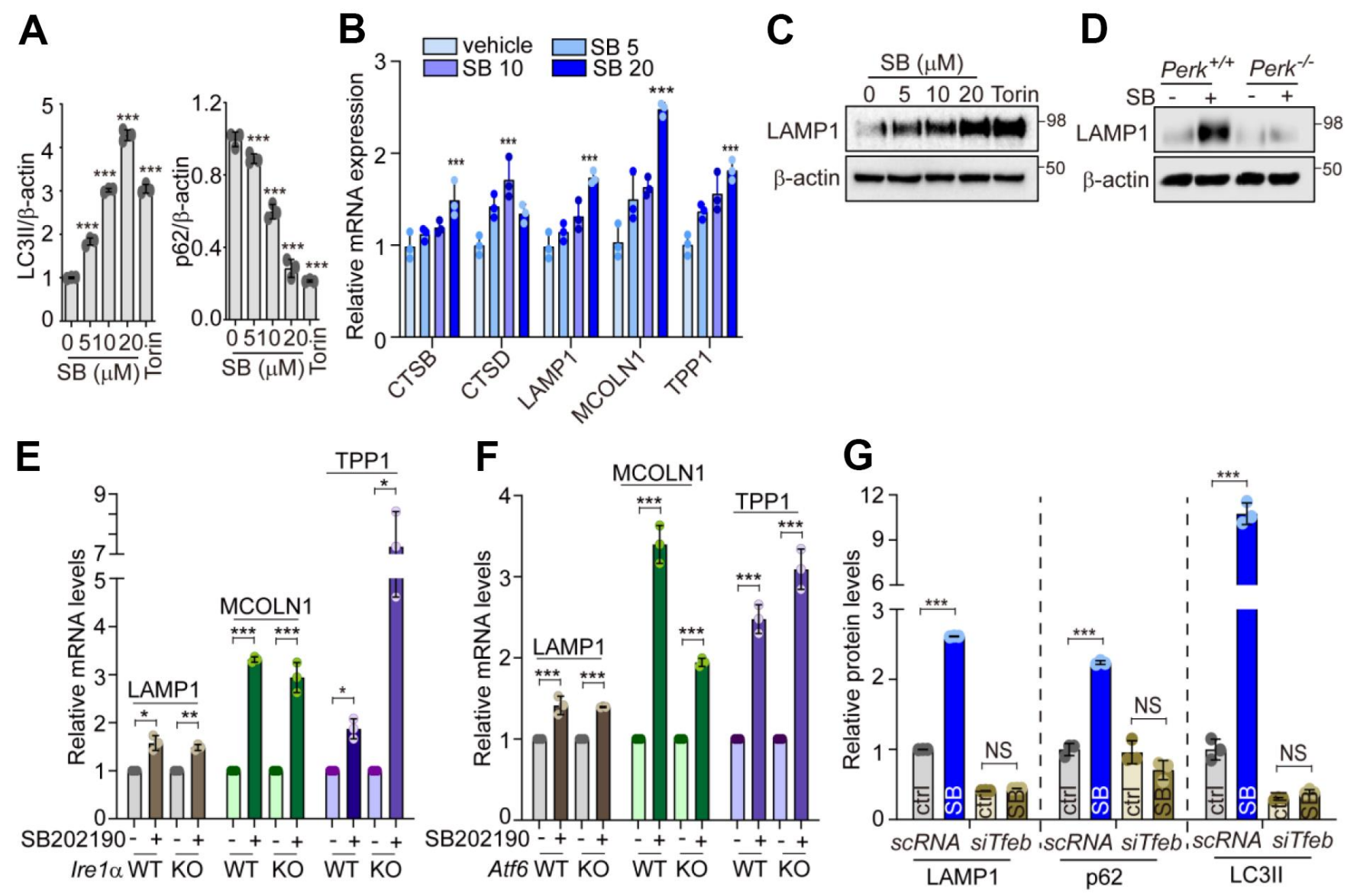

Supplementary Figure 3. PERK activation by SB202190 facilitates autophagy and lysosome biogenesis via TFEB activation related to Figure 5. (A) Western blotting shown in Figure 5A was analyzed to quantify the band densities. Data represent mean \pm SD, ${ }^{* * *} p<0.001$. (B) SH-SY5Y cells were treated with SB202190 $(5,10$, and $20 \mu \mathrm{M})$ for $6 \mathrm{~h}$, and then lysosomal genes were analyzed by qRT-PCR. Data represent mean \pm SD; ${ }^{* * *} p<0.001$. (C) SH-SY5Y cells were treated with SB202190 at the indicated concentrations for 6 h. Torin- $1(2 \mu \mathrm{M})$ was used as a positive control. The levels of LAMP1 and p62 expression were evaluated by western blotting. (D) Perk ${ }^{+/+}$and Perk $/$MEFs were $^{\prime}$ treated with SB202190 for $6 \mathrm{~h}$, and LAMP1 expression was analyzed by western blotting. (E) Western blotting shown in Figure 5E was

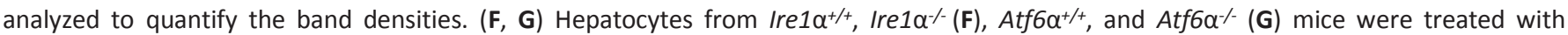
SB202190 $(20 \mu \mathrm{M})$ for $6 \mathrm{~h}$ to assess the levels of LAMP1, MCOLN1, and TPP1 expression by qRT-PCR. Data represent mean \pm SD; ${ }^{*} p<0.05$, ${ }^{* *} p<0.01$, and $* * * p<0.001$. 
A

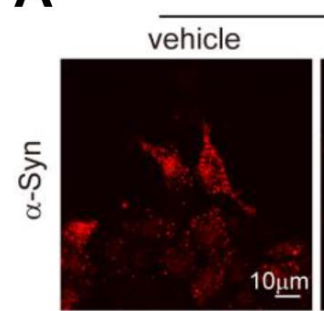
$\alpha$-Syn-A53T

$0 \underline{\underline{\mu}}$
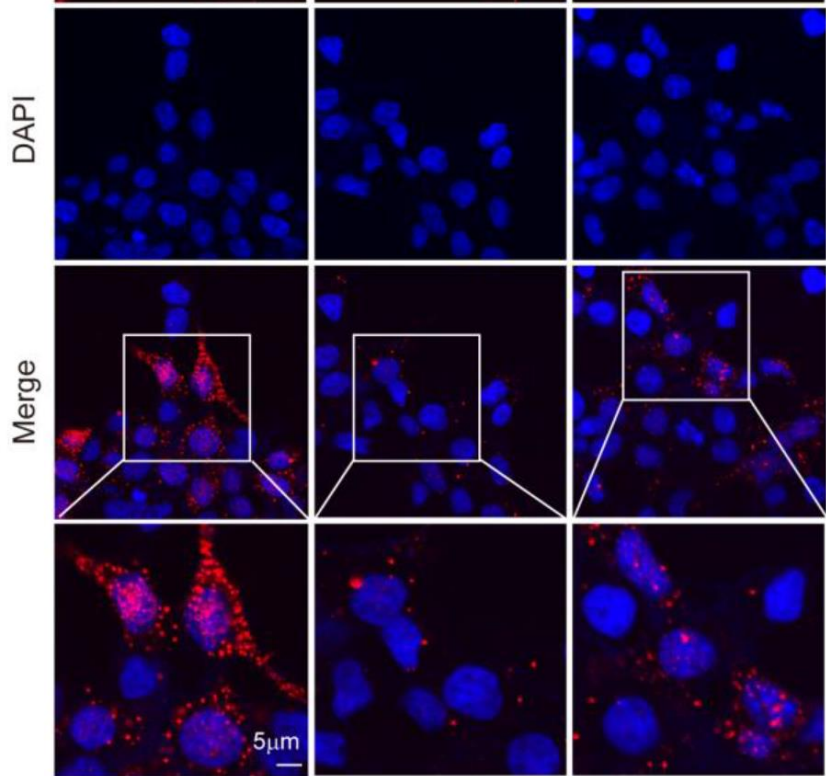

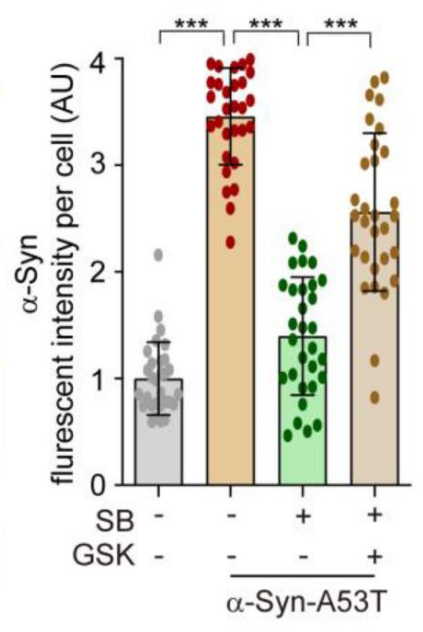

B
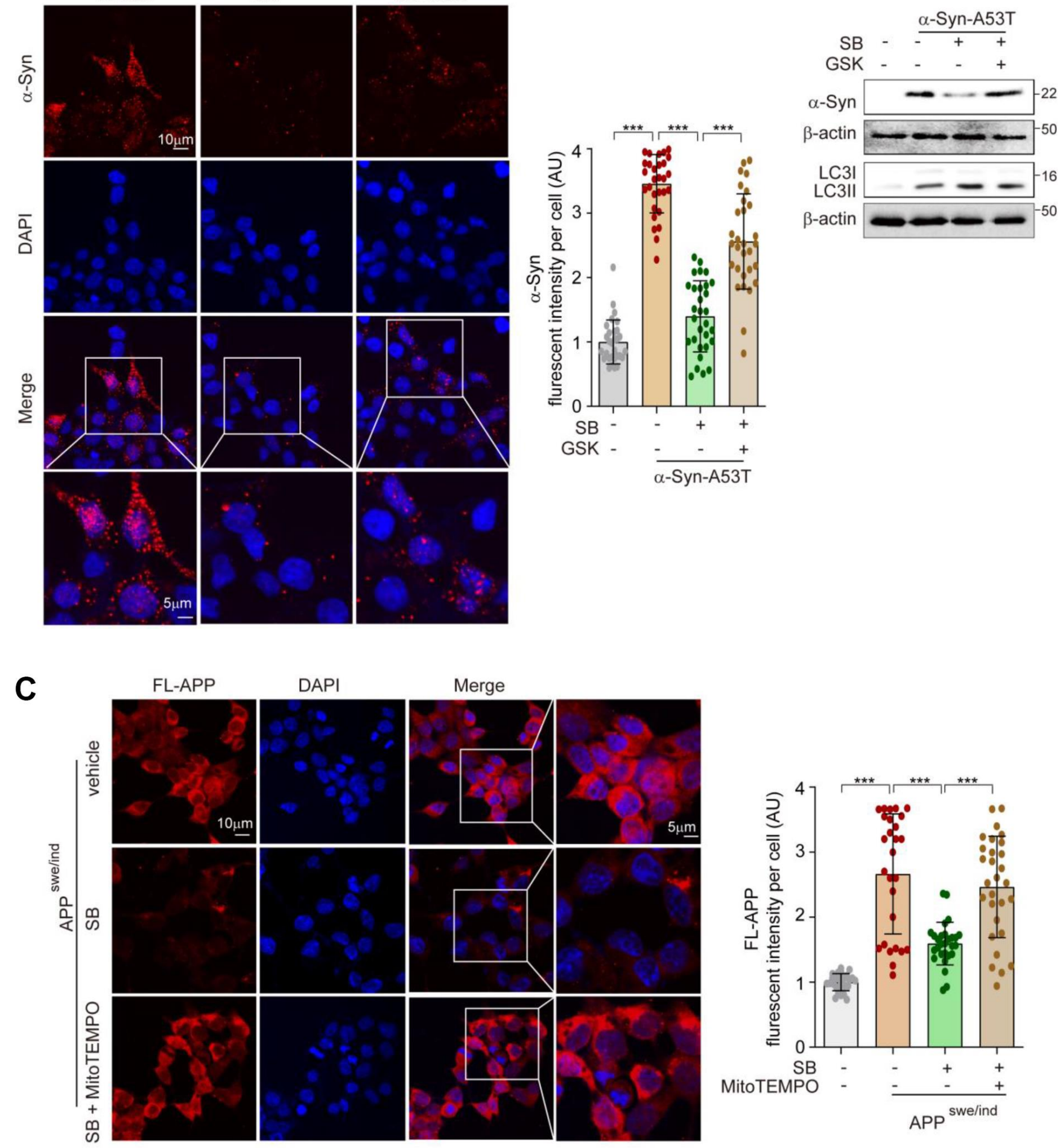

Supplementary Figure 4. PERK activation by SB202190 reduces the aggregation of APP and $\alpha$-syn accumulation through TFEBALP activation in SH-SY5Y cells related to Figure 6. (A, B) SH-SY5Y cells were transiently transfected with $\alpha$-Syn-A53T for $48 \mathrm{~h}$. Cells were treated with GSK2606414 $(1 \mu \mathrm{M})$ for $1 \mathrm{~h}$ before SB202190 $(20 \mu \mathrm{M})$ treatment for $12 \mathrm{~h}$. Representative image of $\alpha$-Syn was detected by confocal microscopy (left) and quantification of $\alpha$-Syn intensity (right). Data represent mean \pm SD; $* * * p<0.001$. (B) The levels of $\alpha$-Syn and LC3B-II conversion were analyzed by western blotting. (C) SH-SY5Y cells were transfected with pCAX-APP-Swe/Ind (APPswe/ind) for 48 h. Cells were subsequently pretreated with MitoTEMPO (100 nM) for $1 \mathrm{~h}$ and then treated with SB202190 $(20 \mu \mathrm{M})$ for $12 \mathrm{~h}$. Representative image of FL-APP was analyzed by confocal microscopy (left) and quantification of FL-APP intensity (right). Data represent mean $\pm \mathrm{SD} ;{ }^{* * *} p<0.001$. 